\title{
Modulation of learning and memory by the targeted deletion of the circadian clock gene $B M A L 1$ in forebrain circuits
}

Kaitlin H. Snider ${ }^{1}$, Heather Dziema ${ }^{1}$, Sydney Aten ${ }^{1}$, Jacob Loeser ${ }^{1}$, Frances E. Norona ${ }^{1}$, Kari Hoyt ${ }^{2}$, Karl Obrietan ${ }^{1}$

${ }^{1}$ Department of Neuroscience, Ohio State University, Columbus, OH, USA

${ }^{2}$ Division of Pharmacology, Ohio State University, Columbus, OH, USA

Corresponding Author:

Karl Obrietan

Department of Neuroscience

Ohio State University

Graves Hall, Rm 4118

333 W. 10th Ave. Columbus, OH 43210

Phone: (614) 292-4432

Fax: (614) 688-8742

E-mail: obrietan.1@osu.edu

(C) 2016. This manuscript version is made available under the Elsevier user license http://www.elsevier.com/open-access/userlicense/1.0/ 


\begin{abstract}
A large body of literature has shown that the disruption of circadian clock timing has profound effects on mood, memory and complex thinking. Central to this time keeping process is the master circadian pacemaker located within the suprachiasmatic nucleus (SCN). Of note, within the central nervous system, clock timing is not exclusive to the SCN, but rather, ancillary oscillatory capacity has been detected in a wide range of cell types and brain regions, including forebrain circuits that underlie complex cognitive processes. These observations raise questions about the hierarchical and functional relationship between the SCN and forebrain oscillators, and, relatedly, about the underlying clock-gated synaptic circuitry that modulates cognition. Here, we utilized a clock knockout strategy in which the essential circadian timing gene BMAL1 was selectively deleted from excitatory forebrain neurons, whilst the SCN clock remained intact, to test the role of forebrain clock timing in learning, memory, anxiety, and behavioral despair. With this model system, we observed numerous effects on hippocampus-dependent measures of cognition. Mice lacking forebrain BMAL1 exhibited deficits in both acquisition and recall on the Barnes maze. Notably, loss of forebrain BMAL1 abrogated time-of-day dependent novel object location memory. However, the loss of BMAL1 did not alter performance on the elevated plus maze, open field assay, and tail suspension test, indicating that this phenotype specifically impairs cognition but not affect. Together, these data suggest that forebrain clock timing plays a critical role in shaping the efficiency of learning and memory retrieval over the circadian day.
\end{abstract}

\title{
Keywords
}

BMAL1, Brain and muscle Arnt-like protein-1, memory, learning, circadian 


\begin{abstract}
Abbreviations
SCN, suprachiasmatic nucleus; BMAL1, Brain and muscle Arnt-like protein-1; CA1, cornu ammonis 1 cell layer of the hippocampus; GCL, granule cell layer of the hippocampus; CaMKII, $\mathrm{Ca}^{2+}$ / calmodulin-dependent protein kinase II; BMAL1 fKO, BMAL1 forebrain knockout; BMAL1 WT, WT-like floxed BMAL1 mice; ZT, zeitgeber time; CT, circadian time; LD, light:dark cycle; DD, total darkness.
\end{abstract}




\section{Introduction}

Work in a wide array of organisms (from Aplysia to humans) has clearly established that learning and memory are modulated by a circadian timekeeper [1-5]. For example, in rodent models, learning tasks such as spatial memory and context and cued fear conditioning vary as a function of circadian time [6-8]. Paralleling these findings, a large number of studies have shown that recall deficits occur as a function of the time of day, indicating that the memory recall process (which is independent of memory formation and acquisition) is also gated by a circadian-dependent process [1,911]. The connection between circadian timing and learning and memory is supported by cellular-based approaches that have shown that underlying physiological properties of the hippocampus are modulated by the clock. Along these lines, clock timing modulates long-term potentiation magnitude in subregions of the hippocampus that include area CA1 (cornu ammonis 1) and the GCL (granule cell layer) [12-14]. Likewise, evoked GCL response properties vary as a function of the circadian cycle [15], and pyramidal cell responsiveness to a range of transmitters (i.e., serotonin, norepinephrine, and acetylcholine) is modulated by the clock [16]. Further, the circadian clock has also been reported to exert control over GABAergic inhibition in the forebrain, a physiological effect that is independent of sleep history (including sleep deprivation [17]). Clearly, circadian variations in electrophysiological signatures of forebrain neurons (both excitatory and inhibitory) could have profound effects on the function of circuits that underlie cognition.

With respect to learning and memory, the functional significance of the master clock located in the suprachiasmatic nucleus (SCN) of the hypothalamus has been described, but the role of the forebrain $\operatorname{clock}(\mathrm{s})$ is not clear. Interestingly, all forebrain regions express the essential clock genes required to generate circadian oscillations [1824], and, indeed, several studies have shown that limbic structures and the cortex 
exhibit low-level rhythmicity [20-24]. Moreover, the activation state of cell signaling pathways involved in learning and memory (such as cAMP/PKA and ERK/MAPK) exhibit daily oscillations in the hippocampus [25]. Further, within the hippocampus, CREB, a transcription factor involved in learning and memory, displays a circadian oscillation in its Serine-133 phosphorylated state $[25,26]$, and germline period1 deletion has been shown to abrogate this rhythm [27]. These findings raise an interesting question: in addition to the SCN, could a critical "time cue" also emanate from the forebrain?

Here, we addressed the role of forebrain clock timing in the modulation of cognition. Using a targeted gene knockout strategy, we show that the disruption of BMAL1 in forebrain excitatory neurons has a detrimental effect on time-of-day regulated learning and memory. These findings suggest that forebrain oscillators work in a coordinated manner with the SCN to shape key aspects of learning and memory as a function of circadian time. 


\section{Materials and Methods}

\subsection{Transgenic Mice}

Three transgenic mouse lines were obtained from Jackson Laboratories. B6.129S4(Cg)-Arntltm1Weit/J (commonly referred to as BMAL1(fl/fl) mice) are homozygous for a floxed allele of the BMAL1 gene. B6.Cg-Tg(Camk2a-cre)T29-1Stl/J mice (commonly referred to as the CaMKII-CRE mouse line) express CRE recombinase driven by the CaMKIIa promoter. B6.129S4-Gt(ROSA)26Sor ${ }^{\mathrm{tm} 1 \text { Sor } / J}$ (here referred to as the ROSA26- $\beta$-gal line), express the $\beta$-galactosidase gene via the CRE-mediated deletion of a floxed stop codon. Mice from the CaMKII-CRE line were interbred with both of the noted floxed lines. To characterize the efficiency and expression pattern of the CaMKII-CRE line, we utilized F1 animals from the CaMKII-CRE::ROSA26 $\beta$-galactosidase cross. To disrupt BMAL1 expression in the forebrain (and to provide a control mouse line), F1 animals from the CaMKII-CRE / BMAL1(fl/fl) cross were bred back into the BMAL1(fl/fl) homozygous mice to produce mice that were homozygous for floxed BMAL1 and either had one copy or did not have a copy of the CaMKII-CRE recombinase transgene. The resulting lines provided us with the targeted knockout of BMAL1 in forebrain excitatory neurons (BMAL1 forebrain knockout animals, hereafter referred to as "BMAL1 $\mathrm{fKO}$ ") and the floxed BMAL1 line (hereafter referred to as "BMAL1 WT") that served as the control, wild-type-like, line. Mice were genotyped as described in [28] for the BMAL1 floxed allele and [29] for the CaMKII-CRE Recombinase transgene. All methods were in compliance with animal use guidelines and approved by the Ohio State University's Institutional Animal Care and Use Committee.

Animals were bred and maintained under a standard $12 \mathrm{~h} / 12 \mathrm{~h}$ Light/Dark (LD) cycle. For tests performed on animals maintained under this light cycle, we use the zeitgeber time (ZT) nomenclature, with ZT0 set as the light-on time, and ZT12 set as the 
time for light-off. For behavioral experiments conducted under ZT conditions, mice were transferred from their LD home cage environment to the testing arena in a lighttight shuttle box and then tested under 10 lux red light. For tests designed to examine behavior under circadian timing conditions, we use the circadian time (CT) nomenclature, with CT0 referring to when light should have been turned on and CT12 used to denote when lights should have been turned off. For these studies, mice were transferred to total darkness (DD) for two days prior to treatment; this eliminates overt effects of light in order to highlight the effects of the endogenous circadian rhythm. Tests conducted under CT conditions also used 10 lux red light.

Behavioral tests utilized three cohorts of mice: one cohort for the novel object location test, another cohort for the locomotor activity and Barnes maze data (wheel running preceded Barnes maze testing), and another cohort of mice that was tested sequentially in the elevated plus maze, the open field assay, and the tail suspension test. Mice were given a minimum of four days of maintenance housing in LD conditions between sequential behavioral tests.

\subsection{Tissue Processing and Immunolabeling}

Procedures were adapted from [30]. Briefly, animals were sacrificed by cervical dislocation and decapitation under ZT conditions. Brain tissue was processed in icecold oxygenated artificial cerebral spinal fluid and cut into 600 micron sections using a vibratome. Sections were fixed in 4\% paraformaldehyde (PFA) for 12 hours. For immunolabeling, we used the Vectastain Elite ABC labeling kit (Vector Laboratories, Burlingame, CA) followed by nickel-intensified 3,3'-Diaminobenzidine (DAB) development (Vector Labs) according to manufacturer directions. The following primary antibodies were used: rabbit anti- $\beta$-galactosidase (1:10,000 dilution; Cortex Biochemicals, San Leandro, CA); rabbit anti-BMAL1 (1:250; AB4140, EMD Millipore, 
Billerica, MA). Bright field images were acquired on a Leica DMIR microscope using Metamorph software (Universal Imaging, Downingtown PA).

\subsubsection{Circadian rhythms and clock resetting}

Analysis of circadian activity rhythms was conducted as in [30]. Briefly, BMAL1 fKO and BMAL1 WT animals were individually housed in running wheel cages, and wheel activity was recorded via a magnetic switch. Light entrainment on a 12 h/12h LD cycle (100 lux) was recorded for 14 days. Next, mice were maintained in total darkness (DD) for 10 days to profile free-running rhythms. For the photic resetting experiment, animals were dark-adapted for 10 days, then exposed to white light (100 lux, 15 min) at CT15, and allowed to run for an additional 10 days. Wheel-running data was collected using VitalView software (Mini Mitter, Bend, OR, USA). Onsets, total activity, and light:dark activity ratios were determined with the assistance of ActiView software (MiniMitter).

\subsubsection{Novel Object Location}

The novel object location task was adapted from [31] and conducted under 5 lux dim red light. Animals were initially dark-adapted (day 0), and then habituated to an arena for 10 minutes (day 1). The arena was $46 \mathrm{~cm}$ x $52 \mathrm{~cm}$ with $30 \mathrm{~cm}$ high walls; black shapes on all four walls provided spatial and contextual cues. Objects (a straight line, corner, plus, or square) were made from LEGO ${ }^{\mathrm{TM}}$ blocks; LEGO ${ }^{\mathrm{TM}}$ blocks allow for the simple creation of shapes of similar size and color that are made from a single type of material (objects shown in Fig. 3B). Object exploration and novel location testing occurred at CT4 or CT16 (24 hours after habituation and after two days of dark adaptation). Essentially, mice explored two LEGO ${ }^{\mathrm{TM}}$ objects in the arena for 5 minutes

(object exploration). Mice were returned to their home cages for a 30 minute rest period. Finally, during the 5 minute test, mice explored the arena with the same two objects: 
one in the same location (familiar) and the other moved to a different location in the arena (novel). Following the initial test, animals were returned to their LD cycle for 4 days. Next, animals were again dark-adapted and underwent habituation, exploration, and testing as above, except at the opposite CT (e.g., if first tested at CT4, the second test was conducted at CT16) and with a different arena context and object set. We used a block design in order to avoid potential order effects: mice were distributed evenly between first testing at CT4 vs first testing at CT16, and between arena/object set 1 first vs arena/object set 2 first. The locations of the objects were randomized for each trial.

Mice were manually scored for the number of seconds spent exploring the novel object and the familiar object. "Exploration" was defined as nose close enough that the mouse's whiskers could reach the object, with clear orientation to the object. Standing or rearing on the object without sniffing was not counted as exploration. Animals that spent less than five seconds total exploration during the test phase were eliminated from analysis.

\subsubsection{Barnes Maze}

Barnes Maze procedures were adapted from [32]. Briefly, mice were tested for spatial memory using a round elevated platform with evenly-dispersed holes around the edges. An escape box was attached to one hole; geometric shapes on the walls surrounding the maze provided spatial references. An electronic metronome (100 beats per minute, 60 decibels at center of maze) was used as an auditory aversive stimulus.

Mice were trained on the Barnes maze at either ZT4 or ZT16. Mice underwent four days of acquisition training on the maze with three trials per day. After acquisition, mice were maintained in their home cages for 17 days (in order to test for long-term memory similarly to [33]). On day 21, mice were returned to the maze for a 90 -second 
probe trial (as utilized in [32]) without an escape box (at the same ZT as the acquisition trials).

For all trials, a mouse was considered to have located the target hole if it entered the escape zone (a donut-shaped area $3 \mathrm{~cm}$ wide that immediately surrounds the escape hole). Performance on the Barnes maze was measured via primary latency (time from the beginning of the trial to when the mouse first entered the escape zone), distance (distance travelled from the beginning of the trial to when the mouse first entered the escape zone), and non-target-quadrant errors (entering the zones of any holes that were not in the target quadrant; this measure counted repeated investigations of a non-targetquadrant hole as additional errors). For all trials, we categorized the search strategy as either spatial (mouse did not re-enter the center of the maze and did not investigate any holes outside of the target quadrant), random (mouse re-entered the center of the maze), or serial (mouse did not re-enter the center of the maze but did visit at least one hole outside of the target quadrant). During acquisition, data from the three trials for each measure were averaged for each mouse to create one data point per mouse per day. For analysis of acquisition search strategy, we calculated the proportion of spatial strategies to non-spatial (random or serial strategies) for each mouse on each day. During the probe trial on day 21, we also quantified the percent of time that the mouse spent in the target quadrant. All measures were scored using Noldus Ethovision XT version 11.5 (Leesburg, Virginia, USA) tracking system and analysis tools.

\subsubsection{Elevated Plus Maze}

The elevated plus maze was conducted either during the subjective day (ZT3-11) or the subjective night (ZT15-23). The elevated plus maze utilized a plexiglass plusshaped maze with four arms, each $5 \mathrm{~cm}$ by $30 \mathrm{~cm}$; the center area was $5 \mathrm{~cm}$ by $5 \mathrm{~cm}$. The maze had white floors, $13 \mathrm{~cm}$ tall black walls on the closed arms, and no walls on the 
open arms. Mice were placed in the center of the maze facing an open arm and allowed to explore for five minutes. The maze was washed between trials with $70 \%$ ethanol.

Total distance travelled and percent time on open arms were scored using Noldus Ethovision XT version 11.5; percent open entries (over total entries) was scored manually from video.

\subsubsection{Open Field Behavioral Assay}

The open field assay was conducted either during the subjective day (ZT3-11) or the subjective night (ZT15-23). It utilized a $46 \mathrm{~cm}$ x $52 \mathrm{~cm}$ off-white arena with $30 \mathrm{~cm}$ high white corrugated plastic walls. Mice were placed in the center of the arena and allowed to explore for five minutes; the surface was cleaned between trials with $70 \%$ ethanol. All measures (immobility, total distance moved, crossing the center, and percent time spent in the center) were scored using Noldus Ethovision XT version 11.5.

\subsubsection{Tail Suspension Test}

The tail suspension test was conducted either during the subjective day (ZT3-11) or the subjective night (ZT15-23). Mice were suspended for six minutes, 36 inches above a counter by using adhesive tape to attach the tail to the edge of a shelf. A rigid plastic tube $(3.5 \mathrm{~cm}$ long, weighing $0.6 \mathrm{~g}$ ) was slipped over the tail to prevent tail climbing. Mice were tested up to three at a time, separated by corrugated plastic sheets. Time spent immobile was tracked using Noldus Ethovision XT version 11.5.

\subsubsection{Visual tracking test}

The visual tracking test was adapted from procedures described in [34]. It utilized a $30 \mathrm{~cm}$ diameter rotating optokinetic response drum with $45 \mathrm{~cm}$ high walls. The walls were covered in $1.5 \mathrm{~cm}$ wide vertical stripes, alternating black and white. Animals were allowed to habituate to the drum for five minutes. Following habituation, 
animals underwent four trials of ten seconds each, during which the drum was slowly turned either right or left. Each head turn was manually scored as "correct" direction (following the direction of the turning drum) or "incorrect" (counter the direction of the turning drum). The score for each mouse was the average correct over total head turns during all of the four trials. All trials were conducted during the subjective day under 500 lux bright white light.

\subsection{Statistics}

Statistics were conducted using IBM SPSS Statistics 22 (Armonk, NY, USA). Twoway ANOVA analysis was used between timepoints and genotypes; repeated measures ANOVA was used when the same mouse was measured more than once (e.g. in the novel object location task). Given the use of a 2x2 design (two genotypes - BMAL1 fKO and BMAL1 WT - by two times of day - day and night), main effects of the two-way ANOVA do not justify the use of post-hoc tests. We used Bonferroni post-hoc tests for $2 \times 2$ tests that showed an interaction effect. Data not dependent on time of day (e.g. correct-direction head turns) were analyzed using independent samples t-tests. All data are shown as mean \pm standard error of the mean (SEM). Power analysis was conducted in $\mathrm{G}^{*}$ Power 3.0.10 [35] and regression analysis was conducted in R 3.1.2.

For the novel object location test, the discrimination index was calculated as [(time spent exploring novel object location - time spent exploring familiar object location) / (time spent exploring novel object location + time spent exploring familiar object location)]. Regression analysis was conducted in $\mathrm{R}$ to confirm that there was no effect of unavoidable factors within the test (experience, e.g. whether the animal was testing for the first or second time on the novel object location test; novel object location, e.g. whether the object was located on the north, east, south, or west side of the arena; and arena / object set, e.g. whether the animal was testing with Context I and objects A 
and B or Context II and objects C and D). Regression analysis used percent exploration as the dependent variable. Where $\mathrm{R}$ regression analysis reported multiple measures of significance (e.g. novel object location is reported as a significance measure for each location), we reported the most significant value. Grubb's test was conducted on novel object location data within each group; one animal (a BMAL1 WT) was removed from the analysis due to being a statistically significant outlier $(p<0.05)$. This removal did not alter which tests achieved significance.

The four days of Barnes maze acquisition utilized a repeated measures two-way ANOVA. Primary latency, distance travelled, and non-target-quadrant errors showed a highly non-normal distribution by Shapiro-Wilks tests of normality $(\mathrm{p}<0.002$ for all three measures on all days), and boxplots indicated a highly right-skewed distribution for these measures (i.e., the majority of data points were low numbers with a few data points being much higher). Thus, primary latency, distance travelled, and non-targetquadrant errors were log transformed before analysis (as in [36]; graphs present raw data). Grubb's test was conducted on Barnes maze acquisition data within each group; two animals (one BMAL1 fKO and one BMAL1 WT) were removed from the analysis due to being statistically significant $(\mathrm{p}<0.05)$ outliers both before and after log transformation. This removal did not alter which tests achieved significance. For the analysis of search strategy on day 21 of the Barnes maze, two-way ANOVA could not be used due to the categorical nature of the data. We instead used Fischer's Exact Test with time-of-day collapsed across groups when we tested for effects of genotype, and genotype collapsed across groups when we tested for effects of time-of-day. 


\section{Results}

\subsection{Conditional forebrain deletion of BMAL1}

To disrupt the canonical core clock transcriptional feedback loop in excitatory neurons of the cortex and limbic system, we employed a targeted gene disruption strategy to disrupt the expression of the BMAL1 gene. BMAL1 forms a heterodimeric transcription factor with Clock (and Npas1), binding to the Ebox element and facilitating the transcription of core clock genes (e.g., Per and $\mathrm{Cry}$ ) as well as clockcontrolled output genes. Hence, BMAL1 is considered a lynchpin of the clock. Indeed, the genetic deletion of BMAL1 (null mice) results in the complete loss of circadian timekeeping [37]. In line with this, the conditional mouse line (generated by Charles Weitz' lab, where loxP sites flank the exon encoding the BMAL1 basic helix-loop-helix (bHLH) [28]) has been used to selectively knockout clock timing in a number of organs, including the retina, liver and pancreas $[28,38,39]$.

For our studies, we crossed the BMAL1 floxed mouse line with a CaMKII-CRE driver line [29] to delete BMAL1 from forebrain excitatory cell populations. The CAMKII driver expresses principally in excitatory forebrain neurons within the central nervous system (CNS) $[50,51]$ and thus would not be expected to disrupt BMAL1 gene expression in peripheral regions, where it is known to play vital roles in regulating metabolism by time of day $[18,38,39]$. Beyond the anatomical and phenotypic specificity of this driver, CaMKII has also been shown to drive transgene expression from several days to several weeks after birth [40-42]. Thus, the time- and cell type-specific deletion of BMAL1 in our model should not alter its expression during early development [4346] nor disrupt its peripheral developmental roles in epidermal stem cells [47], adipocytes [48], and myoblasts [49]. 
Initially, to validate the targeting of CRE recombinase, we crossed the CamKIICRE line to the ROSA26 $\beta$-galactosidase line; hence, the efficacy of CRE-mediated deletion of a floxed stop codon in the ROSA26 $\beta$-galactosidase line was used to profile the brain regions where CRE was expressed. Using immunohistochemical (IHC) labeling against $\beta$-galactosidase, we show that CRE recombinase was expressed in all major forebrain regions, including the hippocampus (Fig. 1A). Importantly, recombination was not detected in the SCN (Fig. 1A).

Next, we validated the successful crossing of the CaMKII-CRE and BMAL1(fl/fl) lines (Fig. 1B) and assessed BMAL1 expression using IHC labeling. Representative data from the CaMKII-CRE::BMAL1(fl/fl) cross (BMAL1 forebrain knockout mice, referred to hereafter as "BMAL1 $\mathrm{fKO}^{\prime \prime}$ ) in Fig. 1C revealed that BMAL1 was deleted from forebrain regions, including the hippocampus and cortex. Importantly, SCN BMAL1 expression was not altered in these mice (Fig. 1C). Of note, a gross histological analysis revealed that the forebrain of BMAL1 fKO mice appeared normal. Further, BMAL1 fKO mice were generated at the expected Mendelian distribution, and the weight of BMAL1 fKO mice were not significantly different $\left(\mathrm{t}_{(10)}=0.559, \mathrm{p}=0.589\right)$ from same-sex BMAL1(fl/fl) littermates that lacked the CaMKII-CRE transgene (referred to hereafter as "BMAL1 WT"). Vision was also assessed as percent correct-direction head tracking in an optokinetic drum, and a strong response was found for both genotypes (Fig. 1D; data shown as box plots of full range; one-way t-tests against a chance response of 50\%, BMAL1 fKO $\left.\mathrm{t}_{(5)}=17.821, \mathrm{p}<0.001, \mathrm{BMAL1} \mathrm{WT} \mathrm{t}_{(10)}=13.970, \mathrm{p}<0.001\right)$. Percent correctdirection head tracking was not significantly different between genotypes $\left(\right.$ Fig. $1 \mathrm{D} ; \mathrm{t}_{(15)}=$ $1.368, \mathrm{p}=0.192)$

To test the idea that the functionality of the SCN clock is conserved in BMAL1 fKO mice, we profiled wheel running activity. Circadian gating of locomotor activity is a well-established read-out of the SCN clock function. Here we provide representative 
double plotted activity graphs of BMAL1 fKO and BMAL1 WT mice (Fig. 2A). BMAL1 fKO mice displayed locomotor activity under LD conditions that was indistinguishable from BMAL1 WT mice. Average total activity (rotations per day) was similar for both genotypes (Fig. 2B; $\mathrm{t}_{(12)}=1.567, \mathrm{p}=0.143$ ). Under a standard 12 hour light/dark cycle, the BMAL1 $\mathrm{fKO}$ exhibited consolidation of wheel running activity to the night time domain, and consolidation was not significantly different from BMAL1 WT mice (mean Light:Dark ratio 0.04334 and 0.03901, respectively, for BMAL1 WT and BMAL1 $\mathrm{fKO;} \mathrm{t}_{(12)}$ $=0.182, \mathrm{p}=0.859)$. Under DD free-running conditions, there was no significant difference in tau between BMAL1 fKO and BMAL1 WT mice $\left(\mathrm{t}_{(12)}=-2.058, \mathrm{p}=0.062\right)$. Further, in dark-adapted mice, the phase-delaying effects of an early night (circadian time 15) light pulse (40 lux, $10 \mathrm{~min}$ ) was not significantly different in BMAL1 fKO mice compared to BMAL1 WT mice (Fig. $\left.2 \mathrm{C} ; \mathrm{t}_{(12)}=0.813, \mathrm{p}=0.431\right)$; hence both lines exhibited a marked phase delay response. Together, these data indicate the SCN clock is functional in the BMAL1 fKO mouse line.

\subsection{Impairment of forebrain-dependent spatial memory}

To test the effects of the deletion of the forebrain clock on circadian rhythms of learning and memory, we utilized the novel object location (NOL) test [31]. NOL tests the memory of the mouse for the location of an object by moving one of two objects (as in Fig. 3A); if the mouse recalls the original locations, it is expected to spend more time exploring the object that has been moved (objects used are shown in Fig. 3B). The NOL test is hippocampus-dependent $[52,53]$ and has been shown to demonstrate a stronger circadian rhythm than the novel object recognition test [31].

In order to examine the effects of time-of-day on performance in the NOL test, we used a balanced crossover design such that half of the animals in each genotype were tested first at CT4 (day) then a week later at CT16 (night), while the other half of 
the animals in each genotype were tested first at CT16 and then a week later at CT4. Novel object recognition has been previously shown to be replicable across multiple trials with the same subjects and novel sets of objects [54,55]. Consistent with this, regression analysis of our results demonstrated that there were no significant confounding effects of the crossover design ( $p>0.10)$; e.g., the performance on the NOL test was not affected by whether it was the first or second time that the animal underwent the test.

In the NOL test, we found an interaction: an effect of genotype that depended on the time-of-day (Fig. 3C, interaction $\mathrm{F}_{(1,15)}=12.499$, $\mathrm{p}=0.003$, repeated measures ANOVA with CT4 and CT16 as repeated measures and genotype as a between-subjects factor). More specifically, BMAL1 WT animals are very similar to BMAL1 $\mathrm{fKO}$ animals at CT4 ( $\mathrm{p}=1.000$, Bonferroni post-hoc test). However, at CT16, BMAL1 WT animals show a significantly higher discrimination index than BMAL1 fKO animals $(\mathrm{p}=0.011$, Bonferroni post-hoc test). Within genotypes, BMAL1 WT animals show a higher discrimination index at CT16 than at CT4 ( $p=0.007$, Bonferroni post-hoc test) while BMAL1 fKO animals show no difference from CT16 to CT4 ( $p=1.000$, Bonferroni posthoc test). Moreover, when comparing the performance of each group and time point to chance levels, only BMAL1 WT animals at CT16 demonstrated above-chance NOL discrimination $\left(\mathrm{t}_{(6)}=9.125, \mathrm{p}<0.0001\right.$, one-sample $\mathrm{t}$-test $)$; the discrimination index of BMAL1 WT animals at CT4, and of BMAL1 $\mathrm{fKO}$ animals at both timepoints, was not different from chance ( $p>0.10$, one-sample t-tests). There was no effect of time-of-day or genotype on total exploration time (Fig. 3D, $p>0.10$, repeated measures ANOVA).

To further test the effects of deletion of the forebrain clock on learning and memory, we utilized the Barnes maze - a circular platform with 20 equally-spaced escape holes, only one of which leads to an escape box. Context cues on the walls surrounding the platform allow the mouse to form a spatial representation of the 
location of the escape box. This is a test of spatial memory that relies in large part on the hippocampus, with a smaller function of nearby medial temporal lobe structures [5658]. Performance on a similar test of spatial memory, the Morris water maze (MWM), has shown circadian variation in rats [7] and diurnal grass rats [33]. To assess potential time-of-day effects of BMAL1 deletion, animals were trained at two circadian time points, either ZT4 (early day, here referred to as "Day") or ZT16 (early night, here referred to as "Night"). Mice were maintained under LD conditions in order to avoid tau drifting that occurs under long periods of total darkness, as well as to avoid confounding effects of phase shifts due to demanding cognitive tasks [7]. Red light was used during training trials to avoid the confounding masking effects that white light has on behavior during the night time domain [30].

At both day and night time points, BMAL1 WT and BMAL1 fKO mice improved their performance over the four days of acquisition training (Fig. $4 ; F_{(2,47)}$ and $p<0.001$ for all measures; acquisition analyzed by two-way ANOVA repeated measures over four days). However, BMAL1 fKO mice trained at both ZT4 and ZT16 took a longer time to find the escape box (Fig. $4 \mathrm{~A} ; \mathrm{F}_{(1,48)}=8.301, \mathrm{p}=0.006$ ), travelled a significantly longer distance to find the escape box (Fig. $4 \mathrm{~B}, \mathrm{~F}_{(1,48)}=10.083$, $\mathrm{p}=0.003$ ), and made significantly more visits to holes not in the target quadrant before locating the escape box (Fig. 4C, $\mathrm{F}_{(1,48)}=12.705, \mathrm{p}=0.001$ ) when compared to BMAL1 WT mice. While there was no effect of ZT on either genotype for primary latency (Fig. $4 \mathrm{~A} ; \mathrm{F}_{(1,48)}=0.575$, $\mathrm{p}=$ 0.453) or distance travelled (Fig. $4 \mathrm{~B} ; \mathrm{F}_{(1,48)}=0.941, \mathrm{p}=0.337$ ), there was a significant effect of ZT on non-target-quadrant errors for both BMAL1 fKO and BMAL1 WT animals (Fig. $\left.4 C ; F_{(1,48)}=5.049, p=0.029\right)$. This is primarily driven by the high number of errors on day 1 in mice trained at ZT16, as the effect of zeitgeber time was not significant for either BMAL1 fKO or BMAL1 WT animals when testing only days two through four (Fig. 4C; $\left.\mathrm{F}_{(1,48)}=2.858, \mathrm{p}=0.097\right)$. Finally, search strategy during acquisition was also altered by 
genotype. The majority of mice in all groups primarily utilized a random strategy on day 1 (a random strategy, defined as having at least one center crossing and at least one non-target quadrant error, was used in $82 \%$ and $86 \%$, respectively, of ZT4 and ZT16 BMAL1 fKO Day 1 trials, and in 83\% and 87\%, respectively, of ZT4 and ZT16 BMAL1 WT Day 1 trials). However, over the four days of acquisition, BMAL1 fKO mice at both ZT4 and ZT16 were significantly less likely to use a spatial strategy (defined as zero non-target-quadrant errors) than BMAL1 WT mice (Fig. 4D; $F_{(1,52)}=8.286, p=0.006$ ). There was no significant effect of ZT on search strategy for either genotype (Fig. 4D; $\left.F_{(1,48)}=3.265, p=0.077\right)$. The effect of genotype on search strategy is illustrated by representative tracks of BMAL1 WT and BMAL1 $\mathrm{fKO}$ animals over the four days of acquisition (Fig. 4E). Overall, BMAL1 fKO mice displayed deficits in acquisition of the Barnes maze regardless of the time of day at which training was conducted.

After acquisition, animals received a 17-day rest period, and then were tested for long-term memory using a probe trial (with no escape box) on day 21; this extended rest period was consistent with the long-term memory assay used by Martin-Fairey and Nunez [33]. We excluded animals that never used a spatial search strategy, as well as animals that had an average primary latency on day 4 of longer than 15 seconds. Thus, animals used in the retention test had exhibited acquisition of the location of the correct hole by day 4. This excluded 13 of 25 (54\%) BMAL1 fKO mice and 8 of 27 (32\%) BMAL1 WT mice. After exclusions, BMAL1 fKO mice trained at both ZT4 and ZT16 spent significantly less time in the correct target quadrant (Fig. 5A, $\mathrm{F}_{(1,27)}=5.838, \mathrm{p}=0.023$ ) and made more non-target-quadrant errors (Fig. 5B, $\mathrm{F}_{(1,27)}=5.588, \mathrm{p}=0.026$ ) than BMAL1 WT mice. Differences between BMAL1 fKO and BMAL1 WT animals at either ZT4 or ZT16 did not reach significance in either time to locate the correct escape hole (Fig. 5C, $\mathrm{F}_{(1,27)}=$ 2.082, $\mathrm{p}=0.161$ ) or in distance travelled to locate the correct escape hole (Fig. 5D, $\mathrm{F}_{(1,27)}=$ 4.122, $\mathrm{p}=0.052)$. However, BMAL1 fKO animals at both ZT4 and ZT16 did use spatial 
search strategies significantly less frequently than BMAL1 WT animals (Fig. 5E, Fischer's Exact Test, $\mathrm{p}=0.024$ ). The differences in search strategy are illustrated by representative tracks of BMAL1 $\mathrm{fKO}$ and BMAL1 WT animal location (Fig. 5F); note the concentration of the BMAL1 WT animal's location in the target quadrant. There was no difference between animals trained at ZT4 and animals trained at ZT16 for both genotypes ( $p>0.100$ for all measures). Thus, in three of five measures, BMAL1 fKO animals that acquired the location of the escape hole by day 4 showed significantly worse recall of the location than BMAL1 WT animals at day 21.

\subsection{Anxiety and mood are unaffected by forebrain BMAL1 knockout}

In addition to circadian gating of learning and memory, there is evidence that circadian rhythms interact with anxiety and behavioral despair (e.g. [59-61]). To test whether or not the forebrain clock influences these behaviors, BMAL1 fKO animals were also tested on the elevated plus maze, the open field assay, and the tail suspension assay. All tests were conducted either during the zeitgeber day (ZT3 to ZT11) or the zeitgeber night (ZT15 to ZT23); thus, animals were maintained under normal LD conditions and tested under dim red light. Two-way ANOVA was used to test for effects of time-of-day and effects of genotype. In the elevated plus maze, there were no significant differences in time-of-day or genotype on distance moved, total entries, percent entries into open arms, and the duration spent in open arms (Fig. 6; $F_{(1,52),} p>$ 0.05). In the open field assay, the BMAL1 fKO animals were similar to the BMAL1 WT mice on all measures at both night and day (Fig. $\left.7 ; F_{(1,60)}, p>0.05\right)$. However, distance moved in the open field was higher during the night for both genotypes (Fig. 7A; $\mathrm{F}_{(1,60)}=$ $15.315, \mathrm{p}<0.001$ ), and mice of both genotypes demonstrated a less anxious phenotype at night, with less time spent immobile (Fig. $7 \mathrm{~B} ; \mathrm{F}_{(1,60)}=12.981, \mathrm{p}=0.001$ ) and more time in the center (Fig. 7C; $F_{(1,60)}=5.441, p=0.023$ ). Frequency of center crossings did not reach significance for time of day in either genotype (Fig. 7D; $F_{(1,60)}=2.649, p=0.109$ ). Finally, 
neither time-of-day nor genotype influenced immobility in the tail suspension test (Fig. $\left.8 ; F_{(1,55)}, p>0.05\right)$. Thus, the impairment of learning and memory observed in the BMAL1 $\mathrm{fKO}$ is a specific behavioral phenotype that does not appear to impact overall mobility, anxiety, or behavioral despair. 


\section{Discussion}

A rich literature across diverse species has demonstrated that the circadian timing system modulates the efficiency of learning and memory $[7,8,62,63]$. In mammals, much of the neuroanatomical work logically has focused on the contribution of the SCN clock to the generation of rhythms in cognition [64-67]. Here, we sought to move this question from the $\mathrm{SCN}$ to ancillary oscillator populations in the forebrain. A rationale for this was based on studies over the past several years showing both that hippocampal neurons generate circadian rhythms [24,68-70], and that hippocampal rhythms in signaling pathways, including the cAMP/PKA and the ERK/MAPK pathway, contribute to rhythms in acquisition and retention [25,27]. With the use of a BMAL1 knockout strategy that allowed for the elimination of clock timing capacity in the forebrain, we were able to selectively interrogate the contribution of the forebrain clock to the daily modulation of cognitive capacity. Our data suggest that forebrain oscillators likely play a key role in circadian modulation of learning and memory.

As noted, in our BMAL1 $\mathrm{fKO}$ model, the SCN is genetically and anatomically intact, and typical circadian locomotor activity is present. However, BMAL1 fKO animals displayed learning and memory deficits similar to those seen in more global models of circadian disruption. Along these lines, the impairments we observed on the NOL test are similar to deficits observed on the novel object recognition test in arrhythmic animals (i.e. the animals are no longer able to perform at better than chance levels [64]). Further, the deficits in Barnes maze performance are similar in magnitude to the impairments seen in BMAL1 knockout mice on the Morris water maze (MWM) [67]; specifically, the mean time to locate the escape box for BMAL1 $\mathrm{fKO}$ animals was $130 \%-190 \%$ of the mean time of BMAL1 WT animals, similar to the mean escape latencies for BMAL1 germline $\mathrm{KO}$ animals in the MWM. If clock gating of learning and memory was only driven by output from the SCN, one might expect to find no 
differences between the BMAL1 fKO and the BMAL1 WT mouse lines; instead, the deficits reported here in forebrain-dependent learning tasks raise the prospect that ancillary clocks in the forebrain may play a critical intermediary role between the master SCN clock and learning and memory.

It is worthwhile to note that the direction of time-of-day differences in the NOL task seen in BMAL1 WT animals is consistent with previous published work. Along these lines, animals have been observed to perform better discrimination at night compared to day in both NOL [31] and in novel object recognition [64]). It is also of interest that while we found that BMAL1 WT mice displayed a marked time-of-day difference on the NOL task, we did not find a time-of-day effect on Barnes maze acquisition and recall. While non-target-quadrant errors in the Barnes maze acquisition assay were significantly higher at ZT16, the fact that this effect is lost when day 1 was not included in the analysis indicates that this is likely a difference in activity or exploratory behavior, not in cognitive ability. Previous research is mixed on the circadian modulation of spatial maze tasks. For example, Gritton et al [7] found circadian modulation on the MWM only during recall and after eight probe trials (i.e. following extinction). In diurnal grass rats, circadian effects were found only during recall but on the first probe trial [33] (i.e. initial recall). However, an experiment on the working memory version of the MWM in rats found a circadian effect on motivation but not memory [71]. One potential explanation for the lack of a time-of-day effect in our Barnes maze data is that the task was not sufficiently challenging to reveal a timeof-day effect. As discussed in Gritton et al [7], modulation of cognition by time-of-day is strongest in cognitively-demanding tasks. Thus, the robust recall of BMAL1 WT animals in the Barnes maze may have obscured the modulatory effects of the circadian clock. 
On the Barnes maze retention test at day 21, it is interesting to note that while significant differences were found for time spent in the target quadrant, non-targetquadrant errors, and search strategy, significance was not reached for distance travelled or primary latency. Other researchers [72-74] have found that spatial learning deficits demonstrated by differences in errors or search strategy do not always extend to distance travelled or latency to reach the escape box. As discussed in [71] and [75], latency is a less sensitive measure of spatial memory than errors. Mice can learn to efficiently search the Barnes maze (reducing distance travelled and primary latency) without having any knowledge of the spatial location of the correct hole. Contrastingly, spatial search strategy and time spent in the target quadrant during a probe trial are measures that can only be optimized by memory of a spatial location. Thus, the statistically significant differences in time spent in the target quadrant, non-targetquadrant errors, and search strategy indicate a specific deficit in spatial memory that does not extend to deficits in efficient search strategies for an unknown location. Thus, while BMAL1 fKO mice were less likely to demonstrate accurate recall of the spatial location of the correct hole, they exhibited an efficient search strategy. This provides further support for the idea that oscillations in the forebrain play a vital role in certain kinds of spatial and contextual memory, but may be less important for task acquisition.

It is relevant to note that because so few BMAL1 $\mathrm{fKO}$ animals reached criteria in Barnes maze acquisition, the numbers of BMAL1 $\mathrm{fKO}$ animals analyzed in the retention trial are low (7 and 5, respectively, at ZT4 and ZT16). However, given the relatively large effect size, post hoc power analysis of a representative measure (percent time in the target quadrant) indicates a power of $67.19 \%$. While this is lower than recommended, it remains higher than many behavioral results published in neuroscience research (extensively reviewed in [76]). For example, a representative meta-analysis of water maze rodent studies [77] had a median power of $18 \%$. Moreover, 
specifically because animals that did not reach acquisition criteria were not analyzed, our long-term retention data is likely to underestimate, rather than overestimate, the deficits in BMAL1 fKO long-term memory. Thus, while some caution should be used in interpreting the deficits in long-term recall, we find it likely that the data presented here represent an accurate and replicable effect of forebrain deletion of BMAL1 on long-term memory.

Our studies did not detect an effect of the forebrain BMAL1 disruption on measures of anxiety or mood. In some respects, an effect of clock disruption on learning without an effect on anxiety or mood is consistent with the work of several other groups. For instance, while Per1 knockout animals show significant deficits in learning, they displayed anxiety levels that were similar to Per1 WT animals [68]. Further, germline BMAL1 knockout animals similarly have impaired learning and memory [67], but did not exhibit elevated levels of anxiety [78]. However, it should be noted that anxiety or depression-like behavior has been reported to occur under constant light conditions (which influence clock timing: $[79,80]$ ), as well as in rodents with combined genetic knockdown of Per1 and Per2 in the nucleus accumbens [61] or knockdown of CLOCK transcription in the ventral tegmental area [60]. Clearly, additional studies will be required to delineate the physiological, neuroanatomical and genetic mechanisms that underlie these effects on mood and affect.

BMAL1 is an essential component of the core molecular clock timing mechanism; in addition to controlling the expression of Per and Cry genes, it also works in a circadian manner to drive the transcription of many clock output genes (e.g. [81]), functions as a cofactor for CLOCK histone acetyltransferase activity [82], and regulates chromatin accessibility [83]. BMAL1 also plays roles in development (including the regulation of visual cortex critical period timing [45], murine epidermal stem cell activity [46], adipogenesis [47], and myogenesis [48]). Thus, we cannot rule out the 
possibility that the disruption of ancillary circadian gene networks or developmental deficits could have contributed to the behavioral effects described here. With this in mind, we do note that the general health of the BMAL1 fKO mice used in this study was not altered. They showed no differences in weight, and there were no gross anatomical differences compared to BMAL1 WT mice.

While disruption of sleep is one potential mechanism by which circadian disruptions can impair cognitive processes (for reviews on sleep and learning see [8486]), in our model it is unlikely that sleep itself played a large role. Support for this idea comes from circadian gene knockout studies where the total amount of sleep was not affected in Per1-Per2 double-knockout mice [87], yet Per1 deletion led to deficits in learning and memory $[27,68]$. Further, in our studies, the activity rhythms of the BMAL1 fKO animals were clearly consolidated with strong subjective night activity and very little activity during the subjective day; a pattern which would be consistent with consolidation of sleep/wake cycles.

While it is unlikely that disruptions of development or sleep are primarily responsible for the learning and memory deficits seen in our model, the involvement of particular molecular mechanisms downstream of BMAL1 are yet to be determined. Oscillations in hippocampal MAPK and cAMP signaling pathways have been shown to play a role in learning and memory [25], as well as oscillations of PER1 [27]. Plasticity is also known to vary as a function of the time of day [88]. Moreover, BMAL1 plays a role in circadian transcription of many clock-controlled genes [45,81], any one of which could play a role in learning and memory. Kyricaou and Hastings [62] propose a model in which the role of circadian rhythms in learning and memory is largely caused by the formation of "transcriptomic nodes" where the expression of many genes are controlled in a circadian manner. Thus, while our data strongly suggest that the learning and memory deficits observed are due to a loss of the ancillary forebrain clock, a 
comprehensive analysis of molecular-level oscillations (or lack thereof) in the BMAL1 fKO remains to be conducted.

While we did not directly test whether forebrain rhythmicity is disrupted in the BMAL1 fKO, recently published work using the same conditional knockout strategy has reported a profound loss of rhythmic gene expression in the targeted tissues, including the liver [39,73], retina [28], and pancreas [38,89]. Further, to our knowledge, BMAL1 has not been found to function in a clock-independent manner in the CNS. These data, coupled with the finding that PER1 rhythms (which are driven by BMAL1) are detected in the cortex at P27 [45], support the idea that the cognitive effects reported here result, at least in part, from the disruption of the clock timing process in the forebrain.

Altogether, our findings suggest that forebrain circadian oscillations are involved in maintaining a permissive environment for memory formation and recall. Roth and Sweat [91] proposed a "time-of-day oscillations" or "reverberations" model to describe the effects of the circadian clock on memory. In line with this idea, it is tempting to speculate that the loss of forebrain timing impairs the ability of excitatory hippocampal cells to sufficiently amplify SCN timing signals, and thus, the rhythmic expression of proteins integral to learning and memory are damped within the forebrain. While the downstream mechanism remains to be elucidated, we have here characterized a functional role for forebrain clocks in cognition. Circadian gating of learning and memory is thus built from a complex circuit involving local clocks in the forebrain, and not merely an output of the master SCN clock. 


\section{Acknowledgements}

This work was supported by the National Institutes of Health (Grant code:

MH103361, NS066345, NS091302) and the National Science Foundation (Grant code:

1354612). The authors have no conflict of interest to declare. We thank Andrew Fischer for assistance with optokinetic assays. 


\section{References}

[1] F.A. Holloway, R.A. Wansley, Multiple retention deficits at periodic intervals after active and passive avoidance learning, Behav. Biol. 9 (1973) 1-14.

[2] R.A. Wansley, F.A. Holloway, Multiple retention deficits following one-trial appetitive training, Behav. Biol. 14 (1975) 135-149.

[3] L.C. Lyons, M. Sol Collado, O. Khabour, C. Green, A. Eskin, The circadian clock modulates core steps in long-term memory formation in Aplysia, J. Neurosci. 26 (2006) 8662-8671.

[4] K.P. Wright, J.T. Hull, C.A. Czeisler, Relationship between alertness, performance, and body temperature in humans, Am. J. Physiol. - Regul. Integr. Comp. Physiol. 283 (2002) R1370-R1377.

[5] T.H. Monk, D.J. Buysse, C.F. Reynolds, S.L. Berga, D.B. Jarrett, A.E. Begley, et al., Circadian rhythms in human performance and mood under constant conditions, J. Sleep Res. 6 (1997) 9-18.

[6] J.A. Davies, V. Navaratnam, P.H. Redfern, A 24-hour rhythm in passive-avoidance behaviour in rats, Psychopharmacologia. 32 (1973) 211-214.

[7] H.J. Gritton, A. Kantorowski, M. Sarter, T.M. Lee, Bidirectional interactions between circadian entrainment and cognitive performance, Learn. Mem. 19 (2012) 126-141.

[8] D. Chaudhury, C.S. Colwell, Circadian modulation of learning and memory in fear-conditioned mice, Behav. Brain Res. 133 (2002) 95-108.

[9] J.P. Hunsicker, R.L. Mellgren, Multiple deficits in the retention of an appetitively motivated behavior across a 24-h period in rats, Anim. Learn. Behav. 5 (1977) 1416.

[10] C. Ko, R. McDonald, M. Ralph, The suprachiasmatic nucleus is not required for temporal gating of performance on a reward-based learning and memory task, Biol. Rhythm Res. 34 (2003) 177-92.

[11] B. Devan, E. Goad, H. Petri, E. Antoniadis, N. Hong, C. Ko, et al., Circadian phaseshifted rats show normal acquisition but impaired long-term retention of place information in the water task, Neurobiol. Learn. Mem. 75 (2001) 51-62.

[12] A. Raghavan, J. Horowitz, C. Fuller, Diurnal modulation of long-term potentiation in the hamster hippocampal slice, Brain Res. 833 (1999) 311-4.

[13] K.M. Harris, T.J. Teyler, Age differences in a circadian influence on hippocampal LTP, Brain Res. 261 (1983) 69-73.

[14] D. Chaudhury, L. Wang, C.S. Colwell, Circadian regulation of hippocampal longterm potentiation, J. Biol. Rhythms. 20 (2005) 225-236. 
[15] C.A. Barnes, B.L. McNaughton, G.V. Goddard, R.M. Douglas, R. Adamec, Circadian rhythm of synaptic excitability in rat and monkey central nervous system, Science. 197 (1977) 91-92.

[16] S. Brunel, C. de Montigny, Diurnal rhythms in the responsiveness of hippocampal pyramidal neurons to serotonin, norepinephrine, gamma-aminobutyric acid and acetylcholine, Brain Res. Bull. 18 (1987) 205-212.

[17] N. Lang, H. Rothkegel, H. Reiber, A. Hasan, E. Sueske, F. Tergau, et al., Circadian modulation of GABA-mediated cortical inhibition, Cereb. Cortex. 21 (2011) 2299_ 2306.

[18] C. Dibner, U. Schibler, U. Albrecht, The mammalian circadian timing system: organization and coordination of central and peripheral clocks, Annu. Rev. Physiol. 72 (2010) 517-549.

[19] L.A. Segall, S. Amir, Exogenous corticosterone induces the expression of the clock protein, PERIOD2, in the oval nucleus of the bed nucleus of the stria terminalis and the central nucleus of the amygdala of adrenalectomized and intact rats, J. Mol. Neurosci. 42 (2010) 176-182.

[20] L.M. Wang, J.M. Dragich, T. Kudo, I.H. Odom, D.K. Welsh, T.J. O’Dell, et al., Expression of the circadian clock gene Period2 in the hippocampus: possible implications for synaptic plasticity and learned behaviour, ASN NEURO. 1 (2009) 139-152.

[21] D. Matsui, S. Takekida, H. Okamura, Molecular oscillation of Per1 and Per2 genes in the rodent brain: an in situ hybridization and molecular biological study, KOBE J. Med. Sci. 51 (2005) 85.

[22] M. Reick, J.A. Garcia, C. Dudley, S.L. McKnight, NPAS2: an analog of clock operative in the mammalian forebrain, Science. 293 (2001) 506-509.

[23] M. Verwey, S. Amir, Variable restricted feeding disrupts the daily oscillations of Period 2 expression in the limbic forebrain and dorsal striatum in rats, J. Mol. Neurosci. 46 (2012) 258-264.

[24] L.E. Chun, E.R. Woodruff, S. Morton, L.R. Hinds, R.L. Spencer, Variations in phase and amplitude of rhythmic clock gene expression across prefrontal cortex, hippocampus, amygdala, and hypothalamic paraventricular and suprachiasmatic nuclei of male and female rats, J. Biol. Rhythms. 30 (2015) 417-36.

[25] K.L. Eckel-Mahan, T. Phan, S. Han, H. Wang, G.C.-K. Chan, Z.S. Scheiner, et al., Circadian oscillation of hippocampal MAPK activity and cAMP: implications for memory persistence, Nat. Neurosci. 11 (2008) 1074-1082.

[26] J. Luo, T.X. Phan, Y. Yang, M.G. Garelick, D.R. Storm, Increases in cAMP, MAPK Activity, and CREB Phosphorylation during REM Sleep: Implications for REM Sleep and Memory Consolidation, J. Neurosci. 33 (2013) 6460-6468. 
[27] O. Rawashdeh, A. Jilg, P. Jedlicka, J. Slawska, L. Thomas, A. Saade, et al., PERIOD1 coordinates hippocampal rhythms and memory processing with daytime, Hippocampus. 24 (2014) 712-723.

[28] K.-F. Storch, C. Paz, J. Signorovitch, E. Raviola, B. Pawlyk, T. Li, et al., Physiological importance of a circadian clock outside the suprachiasmatic nucleus, Cold Spring Harb. Symp. Quant. Biol. 72 (2007) 307-318.

[29] J.Z. Tsien, D.F. Chen, D. Gerber, C. Tom, E.H. Mercer, D.J. Anderson, et al., Subregion-and cell type-restricted gene knockout in mouse brain, Cell. 87 (1996) 1317-1326.

[30] R. Cao, G.Q. Butcher, K. Karelina, J.S. Arthur, K. Obrietan, Mitogen- and stressactivated protein kinase 1 modulates photic entrainment of the suprachiasmatic circadian clock, Eur. J. Neurosci. 37 (2013) 130-140.

[31] Y. Takahashi, K. Sawa, T. Okada, The diurnal variation of performance of the novel location recognition task in male rats, Behav. Brain Res. 256 (2013) 488-493.

[32] K.F. Hansen, K. Karelina, K. Sakamoto, G.A. Wayman, S. Impey, K. Obrietan, miRNA-132: a dynamic regulator of cognitive capacity, Brain Struct. Funct. 218 (2013) 817-831.

[33] C.A. Martin-Fairey, A.A. Nunez, Circadian modulation of memory and plasticity gene products in a diurnal species, Brain Res. 1581 (2014) 30-39.

[34] C. Thaung, K. Arnold, I.J. Jackson, P.J. Coffey, Presence of visual head tracking differentiates normal sighted from retinal degenerate mice, Neurosci. Lett. 325 (2002) 21-24.

[35] F. Faul, E. Erdfelder, A.-G. Lang, A. Buchner, G* Power 3: A flexible statistical power analysis program for the social, behavioral, and biomedical sciences, Behav. Res. Methods. 39 (2007) 175-191.

[36] R.D. Hawkins, T. Cohen, E.R. Kandel, Dishabituation in Aplysia can involve either reversal of habituation or superimposed sensitization, Learn. Mem. 13 (2006) 397403.

[37] M.K. Bunger, L.D. Wilsbacher, S.M. Moran, C. Clendenin, L.A. Radcliffe, J.B. Hogenesch, et al., Mop3 is an essential component of the master circadian pacemaker in mammals, Cell. 103 (2000) 1009-1017.

[38] L.A. Sadacca, K.A. Lamia, A.S. deLemos, B. Blum, C.J. Weitz, An intrinsic circadian clock of the pancreas is required for normal insulin release and glucose homeostasis in mice, Diabetologia. 54 (2011) 120-124.

[39] K.A. Lamia, K.-F. Storch, C.J. Weitz, Physiological significance of a peripheral tissue circadian clock, Proc. Natl. Acad. Sci. 105 (2008) 15172-15177.

[40] J. Pokorný, T. Yamamoto, Postnatal ontogenesis of hippocampal CA1 area in rats. I. Development of dendritic arborisation in pyramidal neurons, Brain Res. Bull. 7 (1981) 113-120. 
[41] J. Pokorný, T. Yamamoto, Postnatal ontogenesis of hippocampal CA1 area in rats. II. Development of ultrastructure in stratum lacunosum and moleculare, Brain Res. Bull. 7 (1981) 121-130.

[42] K.E. Burgin, M.N. Waxham, S. Rickling, S.A. Westgate, W.C. Mobley, P.T. Kelly, In situ hybridization histochemistry of $\mathrm{Ca} 2+/$ calmodulin-dependent protein kinase in developing rat brain, J. Neurosci. 10 (1990) 1788-1798.

[43] L. Gonçalves, M. Vinhas, R. Pereira, T. Pais De Azevedo, F. Bajanca, I. Palmeirim, Circadian clock genes Bmal1 and Clock during early chick development, Dev. Dyn. 241 (2012) 1365-1373.

[44] M. Sládek, A. Sumová, Z. Kováčiková, Z. Bendová, K. Laurinová, H. Illnerová, Insight into molecular core clock mechanism of embryonic and early postnatal rat suprachiasmatic nucleus, Proc. Natl. Acad. Sci. U. S. A. 101 (2004) 6231-6236.

[45] Y. Kobayashi, Z. Ye, T.K. Hensch, Clock genes control cortical critical period timing, Neuron. 86 (2015) 264-275.

[46] M.J. Boden, T.J. Varcoe, A. Voultsios, D.J. Kennaway, Reproductive biology of female Bmal1 null mice, Reproduction. 139 (2010) 1077-1090.

[47] P. Janich, G. Pascual, A. Merlos-Suárez, E. Batlle, J. Ripperger, U. Albrecht, et al., The circadian molecular clock creates epidermal stem cell heterogeneity, Nature. 480 (2011) 209-214.

[48] B. Guo, S. Chatterjee, L. Li, J.M. Kim, J. Lee, V.K. Yechoor, et al., The clock gene, brain and muscle Arnt-like 1, regulates adipogenesis via Wnt signaling pathway, FASEB J. 26 (2012) 3453-3463.

[49] J.L. Andrews, X. Zhang, J.J. McCarthy, E.L. McDearmon, T.A. Hornberger, B. Russell, et al., CLOCK and BMAL1 regulate MyoD and are necessary for maintenance of skeletal muscle phenotype and function, Proc. Natl. Acad. Sci. 107 (2010) 19090-19095.

[50] N.E. Erondu, M.B. Kennedy, Regional distribution of type II Ca2+/calmodulindependent protein kinase in rat brain, J. Neurosci. 5 (1985) 3270-3277.

[51] P.I. Hanson, H. Schulman, Neuronal Ca2+/calmodulin-dependent protein kinases, Annu. Rev. Biochem. 61 (1992) 559-601.

[52] G.R.I. Barker, E.C. Warburton, When is the hippocampus involved in recognition memory?, J. Neurosci. 31 (2011) 10721-10731.

[53] O.Y. Chao, J.P. Huston, S. Nikolaus, M.A. de Souza Silva, Concurrent assessment of memory for object and place: Evidence for different preferential importance of perirhinal cortex and hippocampus and for promnestic effect of a neurokinin-3 R agonist, Neurobiol. Learn. Mem. 130 (2016) 149-158.

[54] J. Besheer, H.C. Jensen, R.A. Bevins, Dopamine antagonism in a novel-object recognition and a novel-object place conditioning preparation with rats, Behav. Brain Res. 103 (1999) 35-44. 
[55] M. Graciarena, A.M. Depino, F.J. Pitossi, Prenatal inflammation impairs adult neurogenesis and memory related behavior through persistent hippocampal TGF 31 downregulation, Brain. Behav. Immun. 24 (2010) 1301-1309.

[56] M. Moscovitch, R.S. Rosenbaum, A. Gilboa, D.R. Addis, R. Westmacott, C. Grady, et al., Functional neuroanatomy of remote episodic, semantic and spatial memory: a unified account based on multiple trace theory, J. Anat. 207 (2005) 35-66.

[57] N.J. Broadbent, L.R. Squire, R.E. Clark, Spatial memory, recognition memory, and the hippocampus, Proc. Natl. Acad. Sci. U. S. A. 101 (2004) 14515-14520.

[58] R.D. Samson, C.A. Barnes, Impact of aging brain circuits on cognition, Eur. J. Neurosci. 37 (2013) 1903-1915.

[59] G. Hampp, U. Albrecht, The circadian clock and mood-related behavior, Commun. Integr. Biol. 1 (2008) 1-3.

[60] S. Mukherjee, L. Coque, J.-L. Cao, J. Kumar, S. Chakravarty, A. Asaithamby, et al., Knockdown of clock in the ventral tegmental area through RNA interference results in a mixed state of mania and depression-like behavior, Biol. Psychiatry. 68 (2010) 503-511.

[61] S. Spencer, E. Falcon, J. Kumar, V. Krishnan, S. Mukherjee, S.G. Birnbaum, et al., Circadian genes Period 1 and Period 2 in the nucleus accumbens regulate anxietyrelated behavior, Eur. J. Neurosci. 37 (2013) 242-250.

[62] C.P. Kyriacou, M.H. Hastings, Circadian clocks: genes, sleep, and cognition, Trends Cogn. Sci. 14 (2010) 259-267.

[63] H. Krishnan, L. Lyons, Synchrony and desynchrony in circadian clocks: impacts on learning and memory, Learn. Mem. 22 (2015) 426-37.

[64] N.F. Ruby, C.E. Hwang, C. Wessells, F. Fernandez, P. Zhang, R. Sapolsky, et al., Hippocampal-dependent learning requires a functional circadian system, Proc. Natl. Acad. Sci. 105 (2008) 15593-15598.

[65] E.L. Zelinski, N.S. Hong, R.J. McDonald, Persistent impairments in hippocampal function following a brief series of photoperiod shifts in rats, Anim. Cogn. 17 (2014) 127-141.

[66] E.A. Van der Zee, R. Havekes, R.P. Barf, R.A. Hut, I.M. Nijholt, E.H. Jacobs, et al., Circadian time-place learning in mice depends on Cry genes, Curr. Biol. 18 (2008) 844-848.

[67] S.M. Wardlaw, T.X. Phan, A. Saraf, X. Chen, D.R. Storm, Genetic disruption of the core circadian clock impairs hippocampus-dependent memory, Learn. Mem. 21 (2014) 417-423.

[68] A. Jilg, S. Lesny, N. Peruzki, H. Schwegler, O. Selbach, F. Dehghani, et al., Temporal dynamics of mouse hippocampal clock gene expression support memory processing, Hippocampus. 20 (2010) 377-88.

[69] S. Amir, V.L. Harbour, B. Robinson, Pinealectomy does not affect diurnal PER2 expression in the rat limbic forebrain, Neurosci. Lett. 399 (2006) 147-150. 
[70] E.W. Lamont, B. Robinson, J. Stewart, S. Amir, The central and basolateral nuclei of the amygdala exhibit opposite diurnal rhythms of expression of the clock protein Period2, Proc. Natl. Acad. Sci. U. S. A. 102 (2005) 4180-4184.

[71] V.S. Valentinuzzi, L. Menna-Barreto, G. Xavier, Effect of circadian phase on performance of rats in the Morris water maze task, J. Biol. Rhythms. 19 (2004) 312324.

[72] K.R. Stover, M.A. Campbell, C.M. Van Winssen, R.E. Brown, Early detection of cognitive deficits in the 3xTg-AD mouse model of Alzheimer's disease, Behav. Brain Res. 289 (2015) 29-38.

[73] S.A. Johnson, A.B. Javurek, M.S. Painter, M.R. Ellersieck, T.H. Welsh, L. Camacho, et al., Effects of developmental exposure to bisphenol A on spatial navigational learning and memory in rats: A CLARITY-BPA study, Horm. Behav. (2015).

[74] G.R. Morel, T. Andersen, J. Pardo, G.O. Zuccolilli, V.L. Cambiaggi, C.B. Hereñú, et al., Cognitive impairment and morphological changes in the dorsal hippocampus of very old female rats, Neuroscience. 303 (2015) 189-199.

[75] T.P. O'Leary, R.E. Brown, Optimization of apparatus design and behavioral measures for the assessment of visuo-spatial learning and memory of mice on the Barnes maze, Learn. Mem. 20 (2013) 85-96.

[76] K.S. Button, J.P.A. Ioannidis, C. Mokrysz, B.A. Nosek, J. Flint, E.S.J. Robinson, et al., Power failure: why small sample size undermines the reliability of neuroscience, Nat. Rev. Neurosci. 14 (2013) 365-376.

[77] Z. Jonasson, Meta-analysis of sex differences in rodent models of learning and memory: a review of behavioral and biological data, Neurosci. Biobehav. Rev. 28 (2005) 811-825.

[78] A.A. Kondratova, Y.V. Dubrovsky, M.P. Antoch, R.V. Kondratov, Circadian clock proteins control adaptation to novel environment and memory formation, Aging. 2 (2010) 285.

[79] J.C. Borniger, Z.D. McHenry, B.A. Abi Salloum, R.J. Nelson, Exposure to dim light at night during early development increases adult anxiety-like responses, Physiol. Behav. 133 (2014) 99-106.

[80] A. Tapia-Osorio, R. Salgado-Delgado, M. Angeles-Castellanos, C. Escobar, Disruption of circadian rhythms due to chronic constant light leads to depressive and anxiety-like behaviors in the rat, Behav. Brain Res. 252 (2013) 1-9.

[81] J.J. McCarthy, J.L. Andrews, E.L. McDearmon, K.S. Campbell, B.K. Barber, B.H. Miller, et al., Identification of the circadian transcriptome in adult mouse skeletal muscle, Physiol. Genomics. 31 (2007) 86-95.

[82] M. Doi, J. Hirayama, P. Sassone-Corsi, Circadian Regulator CLOCK Is a Histone Acetyltransferase, Cell. 125 (2006) 497-508.

[83] J.S. Menet, S. Pescatore, M. Rosbash, CLOCK:BMAL1 is a pioneer-like transcription factor, Genes Dev. 28 (2014) 8-13. 
[84] J.C. Kreutzmann, R. Havekes, T. Abel, P. Meerlo, Sleep deprivation and hippocampal vulnerability: changes in neuronal plasticity, neurogenesis and cognitive function, Neuroscience. 309 (2015) 173-190.

[85] R. Havekes, C.G. Vecsey, T. Abel, The impact of sleep deprivation on neuronal and glial signaling pathways important for memory and synaptic plasticity, Cell. Signal. 24 (2012) 1251-1260.

[86] T.-M. Prince, T. Abel, The impact of sleep loss on hippocampal function, Learn. Mem. 20 (2013) 558-569.

[87] P.J. Shiromani, M. Xu, E. Winston, S. Shiromani, D. Geraschenko, D. Weaver, Sleep rhythmicity and homeostasis in mice with targeted disruption of mPeriod genes, AJP Regul. Integr. Comp. Physiol. 287 (2004) R47-R57.

[88] R. Iyer, T.A. Wang, M.U. Gillette, Circadian gating of neuronal functionality: a basis for iterative metaplasticity, Front. Syst. Neurosci. 8 (2014) 164.

[89] M. Perelis, B. Marcheva, K. Moynihan Ramsey, M.J. Schipma, A.L. Hutchison, A. Taguchi, et al., Pancreatic cell enhancers regulate rhythmic transcription of genes controlling insulin secretion, Science. 350 (2015) aac4250-aac4250.

[90] M. Izumo, M. Pejchal, A.C. Schook, R.P. Lange, J.A. Walisser, T.R. Sato, et al., Differential effects of light and feeding on circadian organization of peripheral clocks in a forebrain Bmal1 mutant, eLife. 4 (2015) e04617.

[91] T. Roth, J. Sweatt, Rhythms of Memory, Nat. Neurosci. 11 (2008) 993-994. 


\section{Figure Legends}

Figure 1: Generation of the BMAL1 forebrain knockout mouse line. For this study, we used a CaMKII driver line to target CRE recombinase to forebrain excitatory neurons. (A) To validate this targeting approach, we crossed the CaMKII CRE line to the ROSA26 $\beta$-galactosidase reporter line, and CRE-mediated gal expression was examined via immunohistochemical (IHC) labeling. Of note, $\beta$-galactosidase transgene expression was detected in the CA1 neuronal cell layer, but not in the $\mathrm{SCN}$, thus indicating that the driver expresses as expected: in excitatory forebrain neurons. (B) PCR validation of the successful generation of the CaMKII-CRE::BMAL1 floxed (fl/fl) mouse line. Note that mouse 2 has both two floxed copies of the BMAL1 allele and the CRE transgene. (C) Representative IHC labeling for BMAL1 in CaMKII-CRE control mice (CRE::BMAL1 $(+/+))$ and CRE::BMAL1 (fl/fl) mice. Forebrain BMAL1 labeling in the cortex (CTX) and granule cell layer of the hippocampus (GCL) was disrupted in the BMAL1 (fl/fl) mice. Of note, the CaMKII CRE driver does not delete in all forebrain cells, only in excitatory neurons. In line with this, expression of BMAL1 in the CRE::BMAL1 (fl/fl) mice was still detected in non-neuronal cells and in interneurons. Arrows denote BMAL1 expression in what appears to be non-neuronal cells in the cortex and in the neurogenic niche within the subgranular zone (sgz) within the dentate gyrus. Importantly, BMAL1 expression in the SCN was not affected in the BMAL1 (fl/fl) mice. Together, these data indicate targeted deletion of BMAL1 was achieved. (D) To test for visual acuity, mice were tested for correct head turns using an optokinetic drum. Similar acuity was measured between the genotypes, and responses were significantly different from chance head turning level (50\%) which is denoted by a black line horizontal line. Data are presented as boxplots of the range (box indicates $2^{\text {nd }}$ and $3^{\text {rd }}$ quartiles, error bars indicate highest and lowest data points); $\mathrm{n} 7$ to 11 mice per group; n.s. $\mathrm{p}>0.05$. 
Figure 2: Circadian activity rhythms in BMAL1 fKO mice. (A) Representative doubleplotted actograms of running wheel activity for a BMAL1 WT (BMAL1(fl/fl) mouse) and a BMAL1 fKO (CaMKII-CRE::BMAL1(fl/fl) mouse). Bars across the top designate light periods (white bars) or dark periods (black bars) during the LD period. After 10 days of LD conditions, mice were transitioned to constant darkness (DD), designated by the gray background. The arrows designate a light pulse (15 min, 40 Lux) administered at CT15 in order to induce a phase delay. Regression lines were added to activity onsets to denote the phase-delaying effects of the light pulse. (B) Mean wheel-running activity (rotations per day) over five days in LD conditions was similar for both genotypes. (C) Mean phase delay in response to a CT15 light pulse was similar for both genotypes. Data are presented as the mean \pm SEM phase delay; $n=6$ to 8 mice per group; $n . s . p>$ 0.05 .

Figure 3: Time-of-day dependent learning using the novel object location (NOL) test. (A) Experimental design: mice were first allowed to explore an arena with two objects placed in reference to spatial cues on the arena walls (Explore). After a thirty minute delay, mice were returned to the arena (Test); one object was in the same location (Familiar), while the other object had been moved (Novel). (B) Images of the LEGO ${ }^{\mathrm{TM}}$ objects used in the NOL test. (C) Discrimination index of BMAL1 fKO and BMAL1 WT mice at circadian day (CT4) and circadian night (CT16); calculated as [(Novel Familiar) / (Novel + Familiar)]). (D) Total exploration time of BMAL1 fKO and BMAL1 WT mice at circadian day (CT4) and circadian night (CT16). Data are presented as the mean \pm SEM and were analyzed by repeated measures ANOVA with Bonferroni posthoc tests; $\mathrm{n}=7$ to 10 mice per group; ${ }^{*} \mathrm{p}<0.05$.

Figure 4: Barnes Maze acquisition. (A) Mean primary latency (i.e., seconds to reach the escape box) for the four days of acquisition are presented for mice trained at ZT4 or ZT16. Of note, BMAL1 fKO mice took significantly longer to find the escape box 
compared to BMAL1 WT mice at both circadian times. (B) Distance traveled to reach the escape hole during the four days of acquisition for mice trained at ZT4 or ZT16. BMAL1 fKO mice travelled significantly longer distances compared to BMAL1 WT mice at both circadian times. (C) Non-target-quadrant errors during the four days of acquisition, for mice trained at ZT4 or ZT16. BMAL1 fKO mice made significantly more errors than BMAL1 WT mice at both circadian times; mice trained at ZT16 made significantly more errors than mice trained during ZT4 for both genotypes. (D) Proportional representation of search strategies used by mice over the four days of acquisition; Spatial strategy indicates zero non-target-quadrant errors; Random indicates at least one non-target error and at least one center crossing; Serial indicates at least one non-target error but zero center crossings). BMAL1 WT mice used spatial strategies significantly more frequently than BMAL1 fKO mice. (E) Representative tracks of ZT4 mice prior to finding the escape box during days 1 and 3 of acquisition. Arrows indicate the escape box locations; lighter gray is used to denote the locations of the target quadrants. Data are presented as the mean $\pm S E M$; analyzed by repeated measures two-way ANOVA; $\mathrm{n}$ $=12$ to 15 mice per group; ${ }^{*} \mathrm{p}<0.05$.

Figure 5: Long-term retention of spatial memory in the Barnes maze. (A) Percent time spent in the target quadrant on the day 21 probe trial. BMAL1 fKO mice spent significantly less time in the target quadrant compared to BMAL1 WT mice at both circadian times. (B) Non-target-quadrant errors for the day 21 probe trial. BMAL1 fKO mice made significantly more errors than BMAL1 WT mice at both circadian times. (C) Primary latency (seconds to reach the escape box) on the day 21 probe trial. Difference between genotypes did not reach significance. (D) Distance to reach the escape hole during the day 21 probe trial. Difference between genotypes did not reach significance. (E) Proportional representation of search strategies used by mice on the day 21 probe trial (see figure legend 3 for a description of each strategy). BMAL1 WT mice used 
spatial strategies significantly more frequently than BMAL1 fKO mice. F) Heatmaps of mouse locations during the day 21 probe trial. Arrows indicate the escape box locations; the target quadrants are outlined. Data are presented as the mean $\pm \mathrm{SEM} ;(A)$ through (D) analyzed by two-way ANOVA, (E) analyzed by Fischer's Exact Test; $\mathrm{n}=5$ to 10 mice per group; ${ }^{*} \mathrm{p}<0.05$, n.s. $\mathrm{p}>0.05$.

Figure 6: Elevated plus maze. (A) Mean total distance traveled and, (B) mean total arm entries were not different between BMAL1 WT and BMAL1 fKO mice. (C) Mean percent open entries (open entries / total entries), and (D) mean duration spent in the open arms (s) were also not different between groups. Data are presented as the mean \pm SEM; analyzed by two-way ANOVA; $\mathrm{n}=11$ to 17 mice per group; $n . s . \mathrm{p}>0.05$.

Figure 7. Open field assay. (A) Mean total distance moved was significantly higher in the subjective night for both WT and fKO mice. (B) Mean immobility (seconds: s) was significantly lower in the subjective night for both WT and fKO mice. (C) Mean time spent in the center of the open field was significantly higher in the night for both WT and fKO mice. (D) Mean crossings of the center of the open field; the difference between night and day crossings did not reach significance for both WT and fKO mice. Data are presented as the mean \pm SEM; analyzed by two-way ANOVA; $n=14$ to 19 mice per group; ${ }^{*} \mathrm{p}<0.05$, n.s. $\mathrm{p}>0.05$.

Figure 8. Tail suspension test. These data reveal a similar level of behavioral despair in both genotypes and at both times of day. Data are presented as the mean time-spent immobile (seconds: s) during the tail suspension test. Data are presented as the mean \pm SEM; analyzed by two-way ANOVA; $\mathrm{n}=13$ to 16 mice per group; n.s. $\mathrm{p}>0.05$. 
A

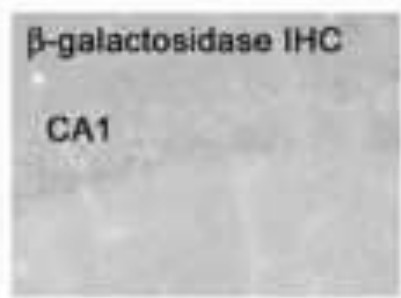

CaMKII-CRE:ROSA26 $\beta$-Gal

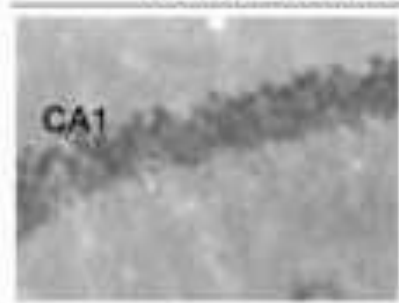

CaMKII-CRE
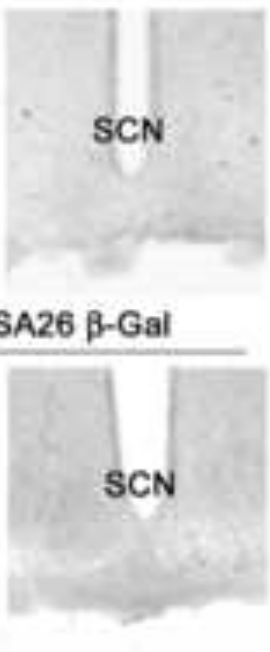

B

\section{CaMKII-CRE:BMAL1 $1(x / x)$}
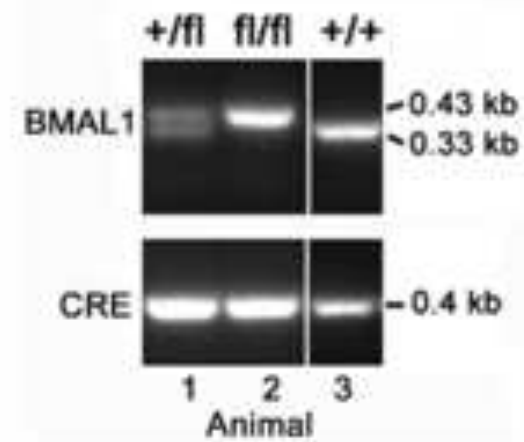

\section{C}

BMAL1 WT [CaMKIL-CRE::BMAL1(+/+)]
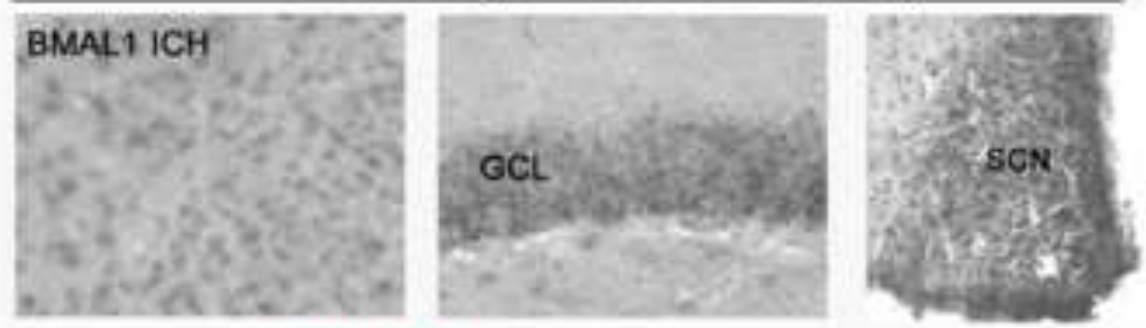

BMAL1 fKO [CaMKH-CRE::BMAL_(fI/f)]

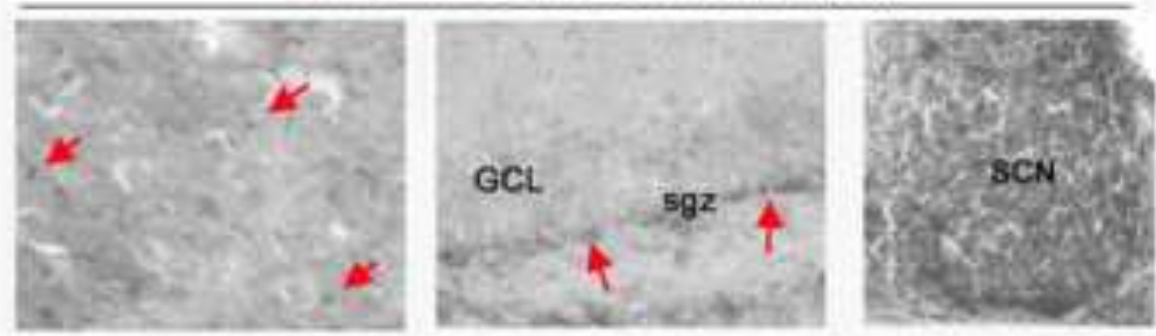

D

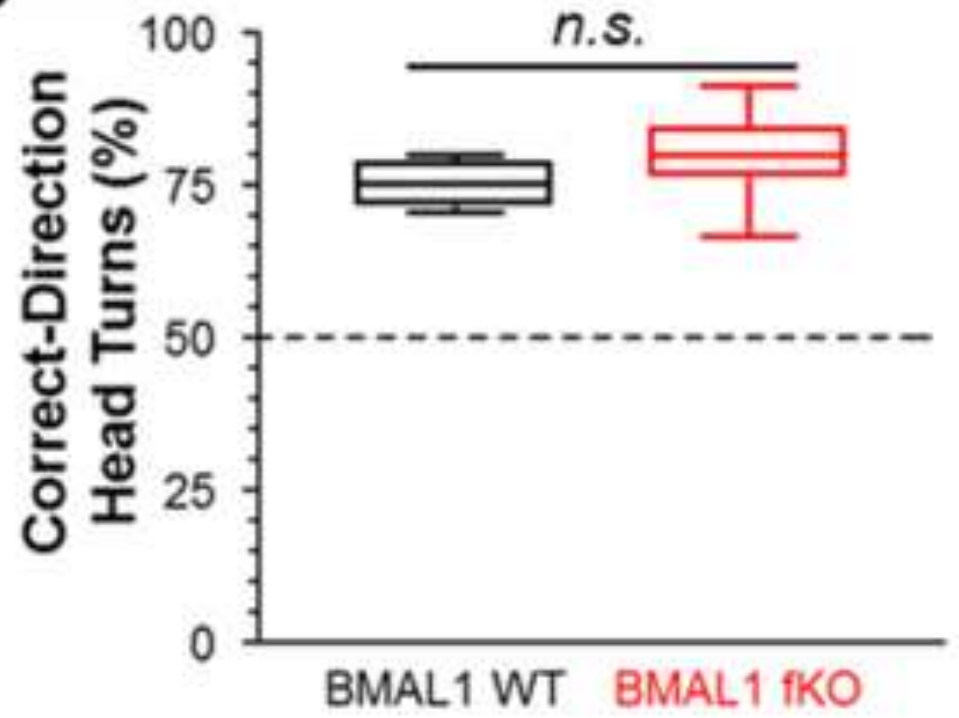


A

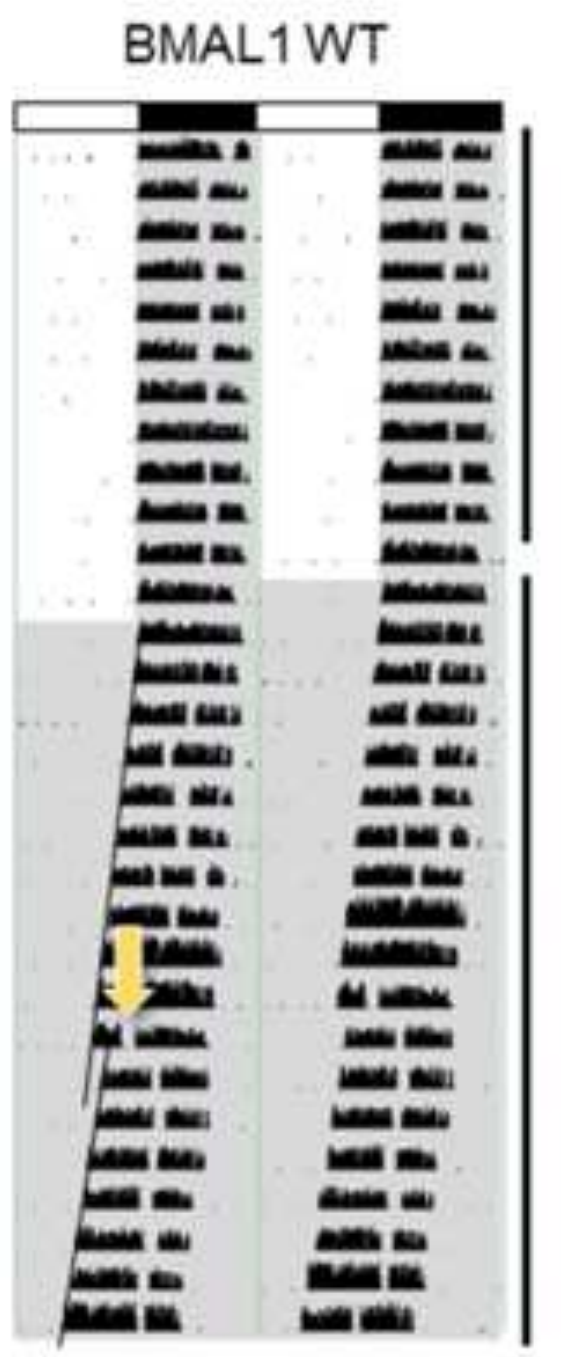

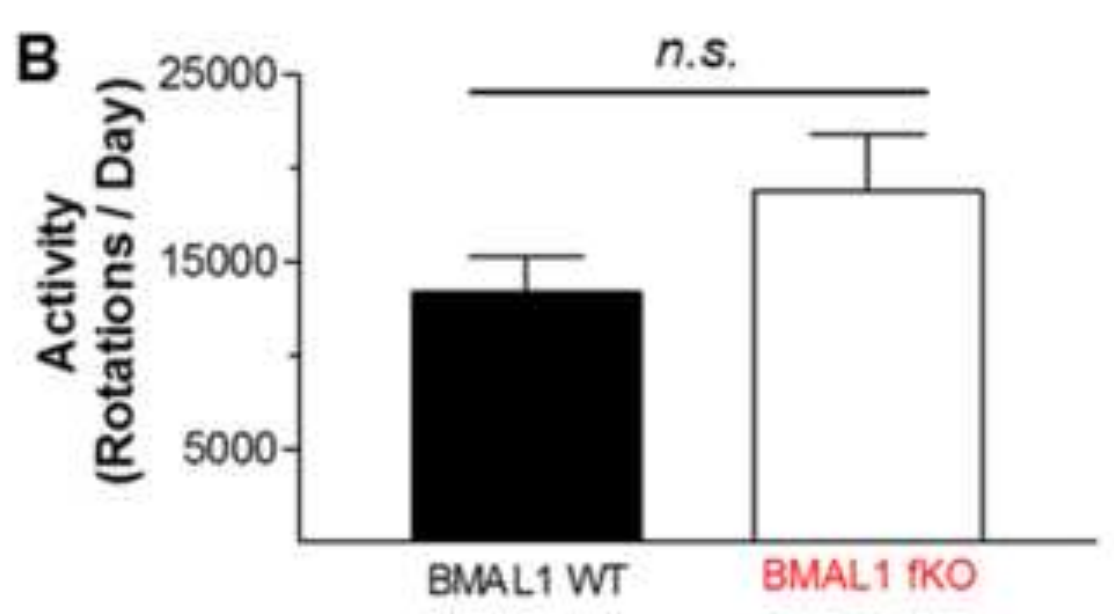

C

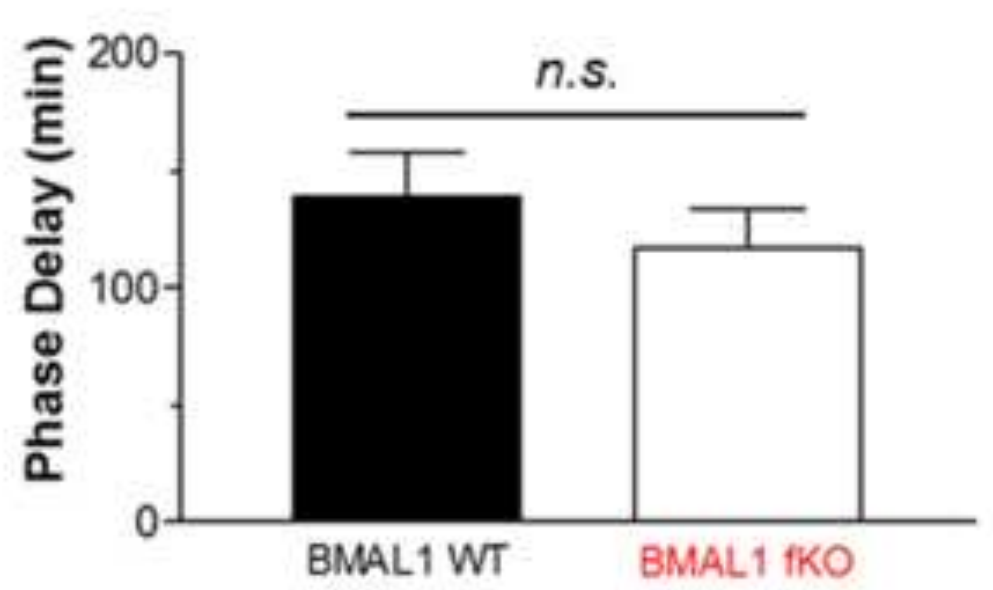


Figure 3

A

Explore

Test

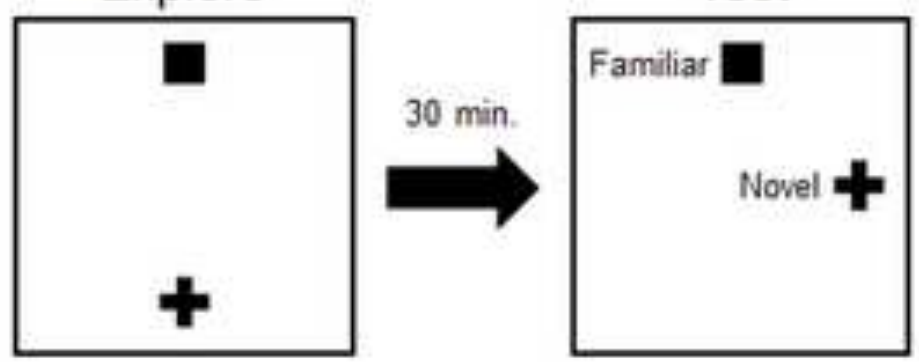

B

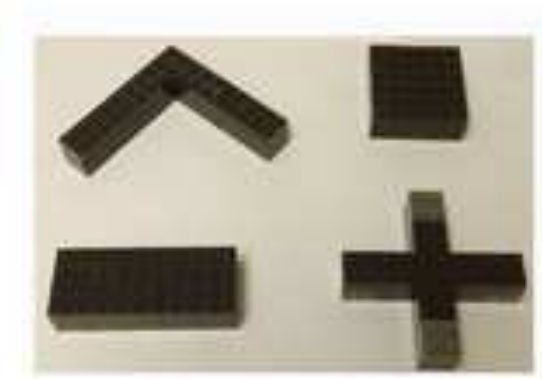

C

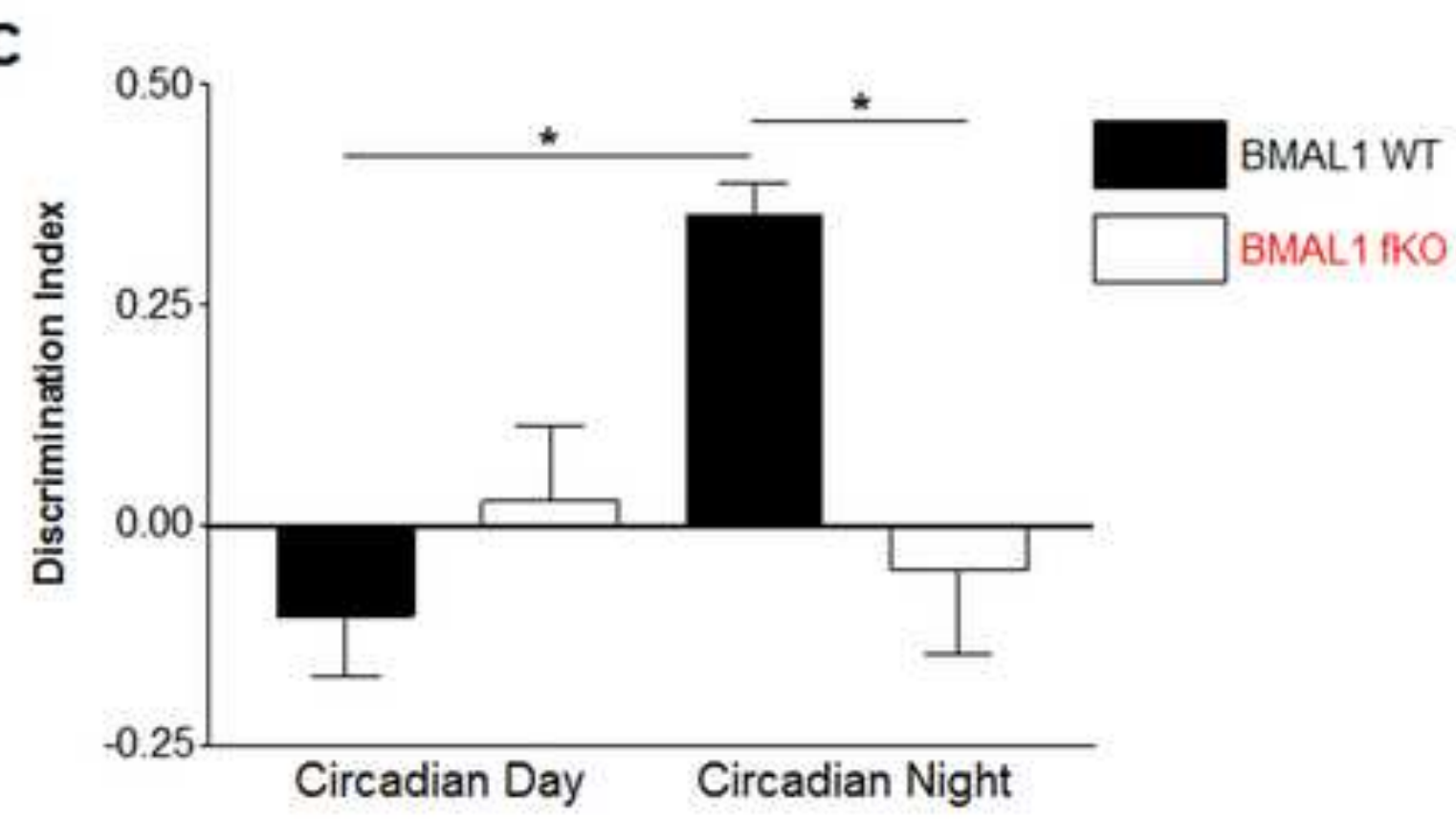

D

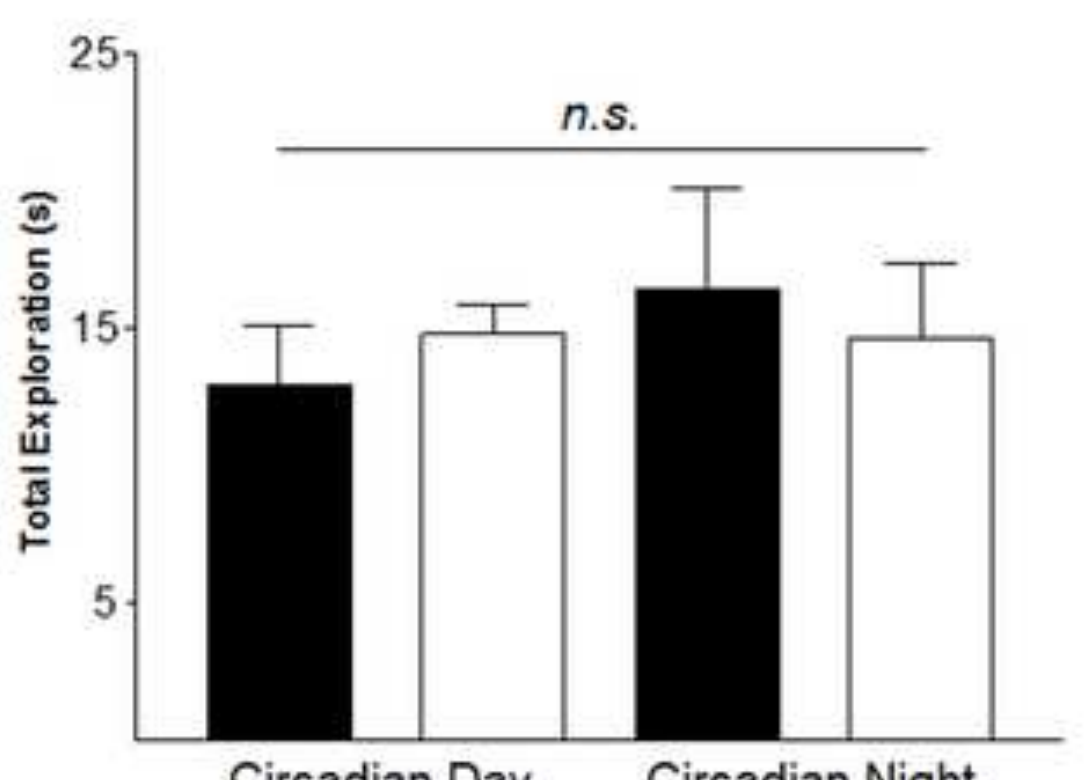

Circadian Day Circadian Night

D 

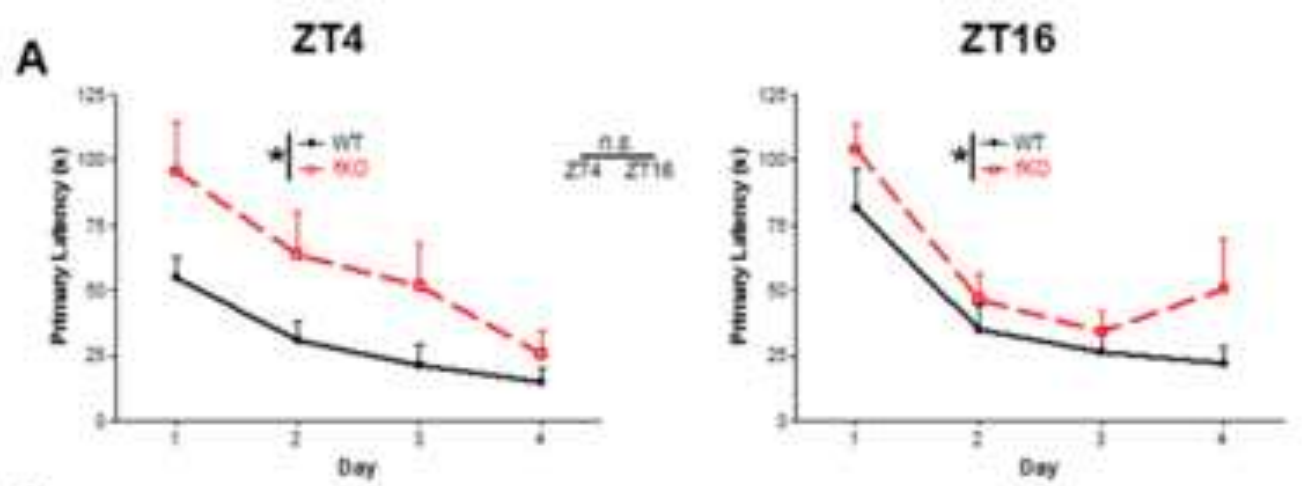

D
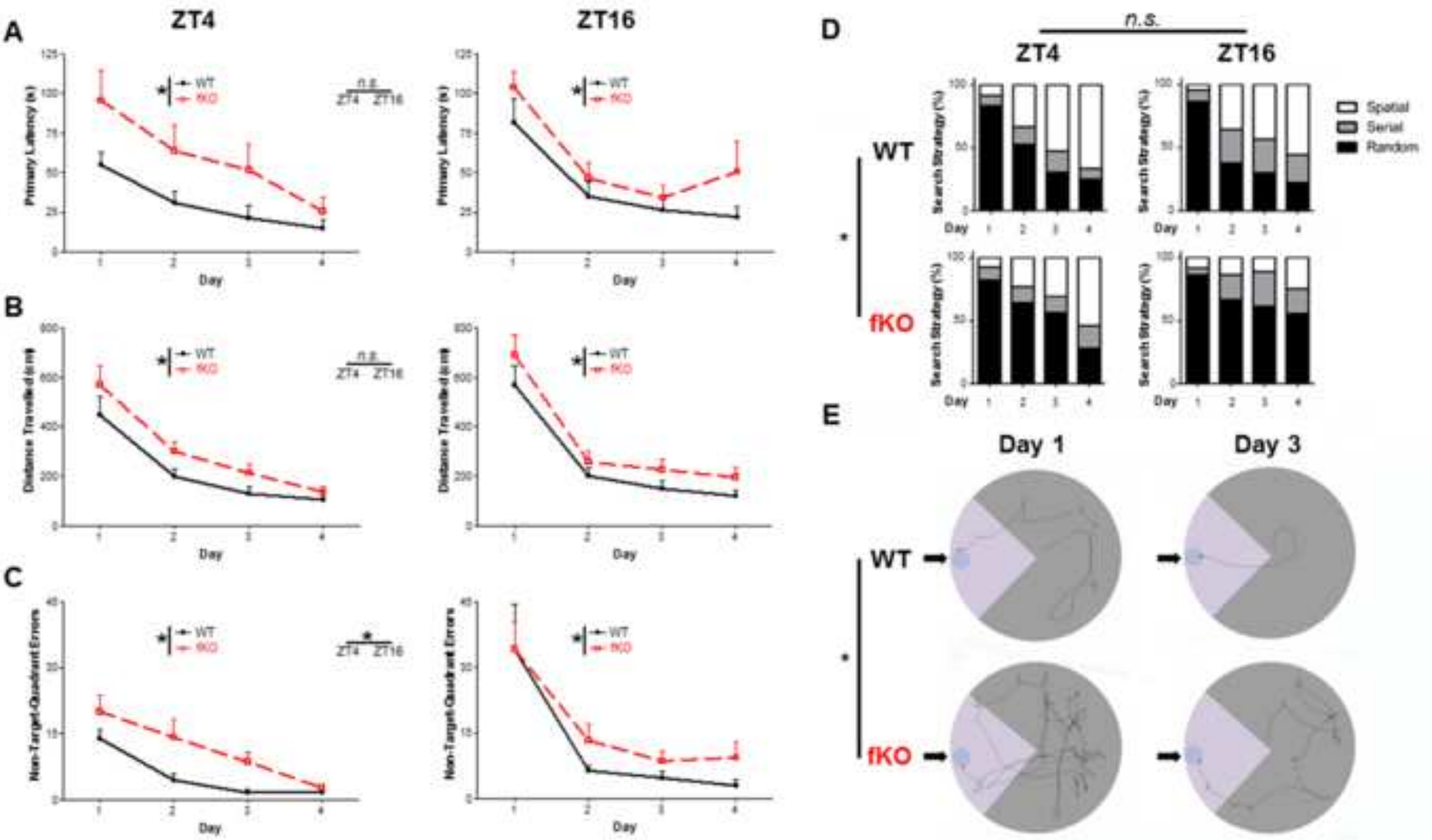

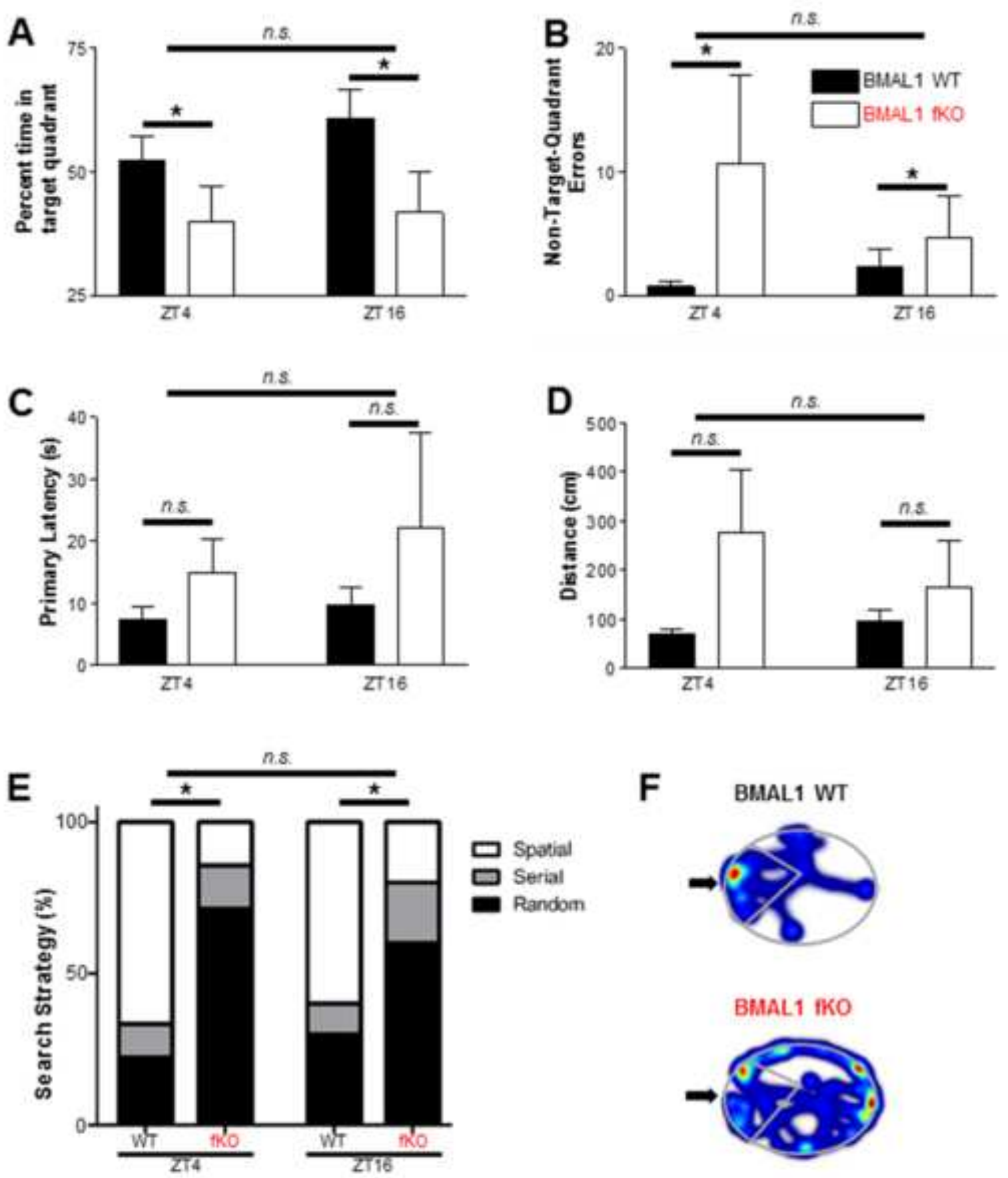
n.s.

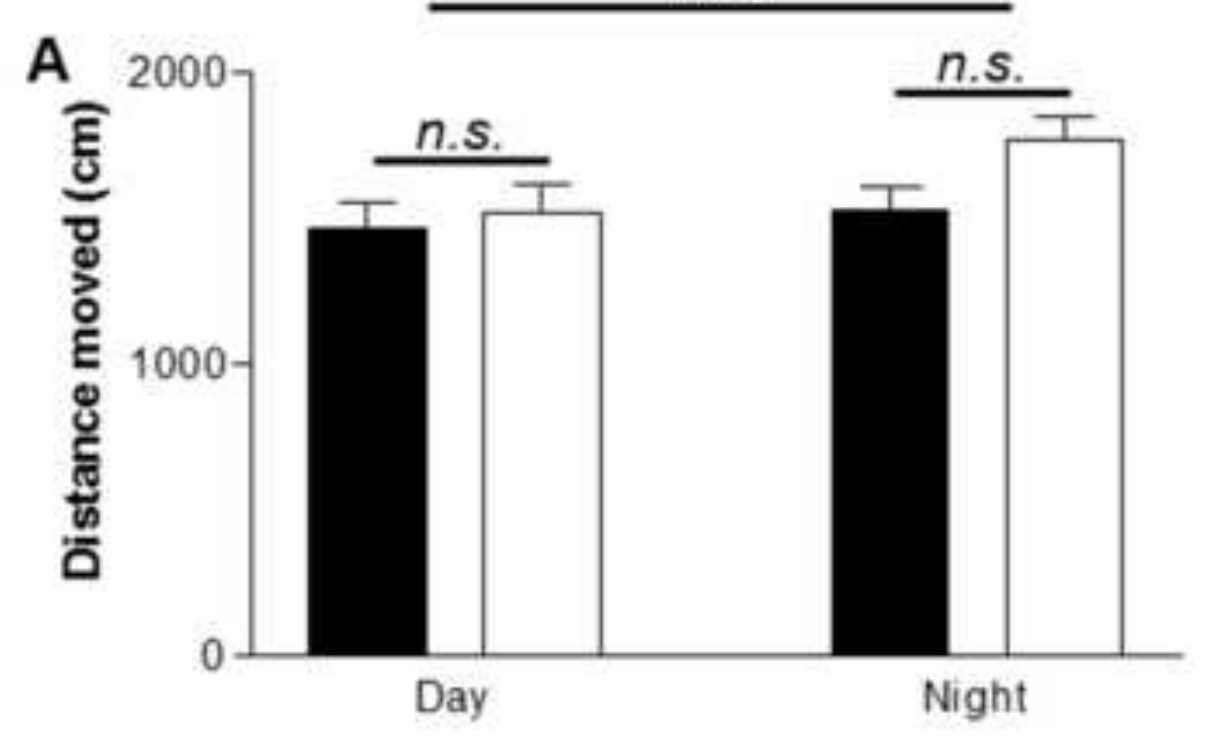

C

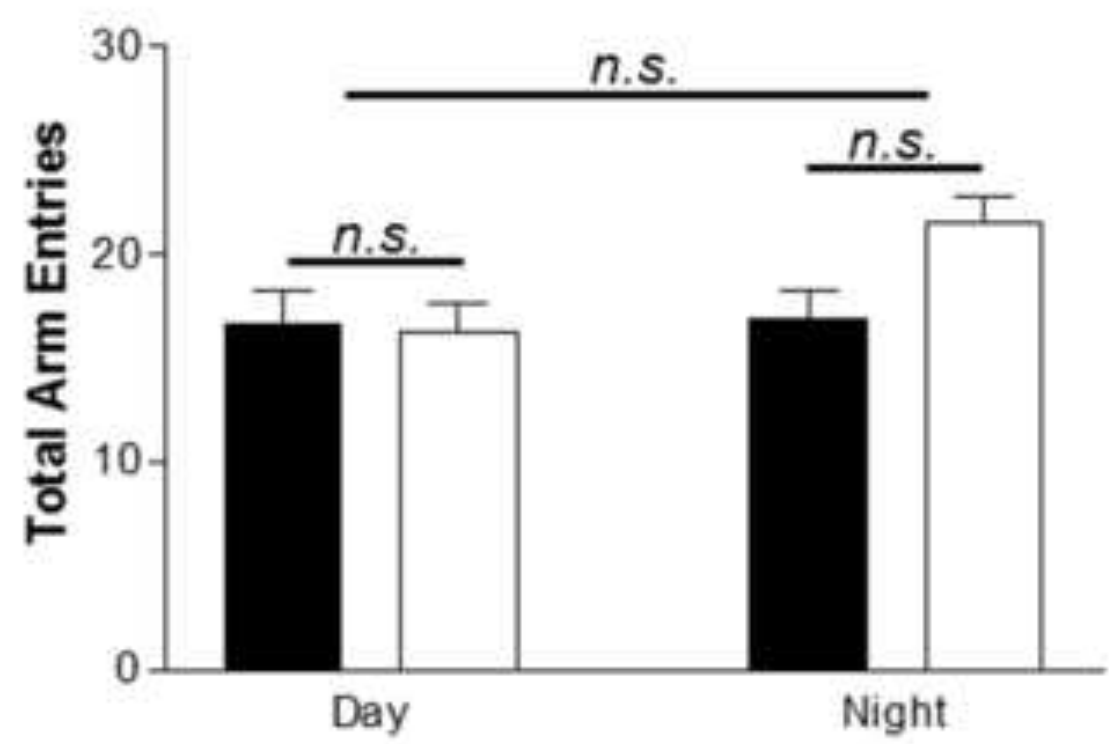

B

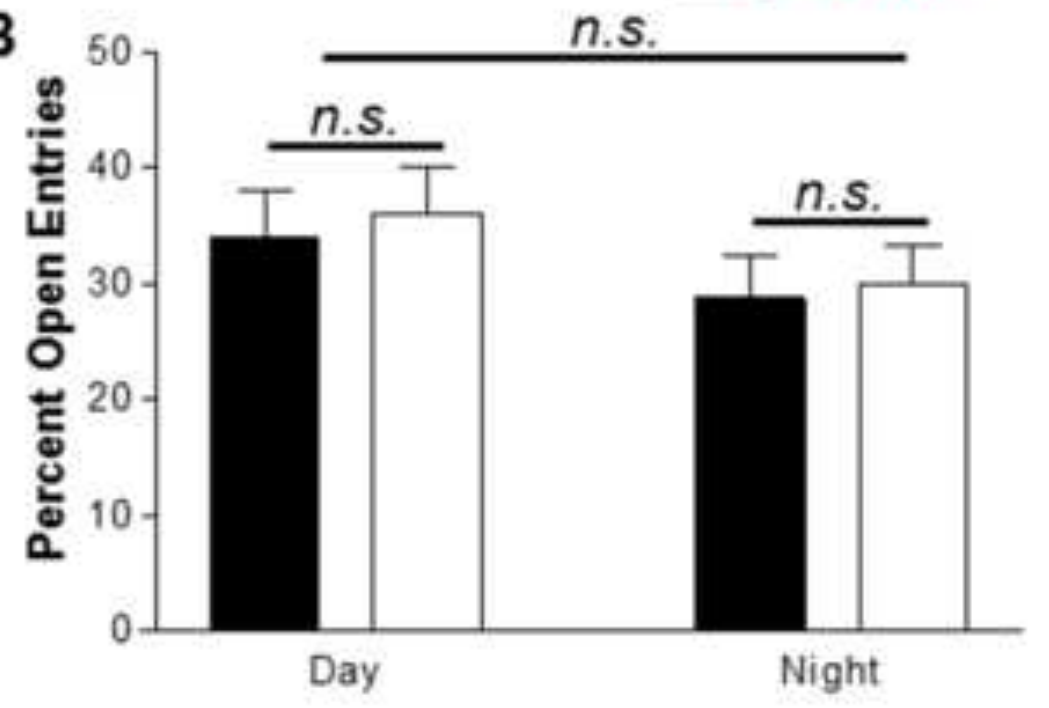

n.s.

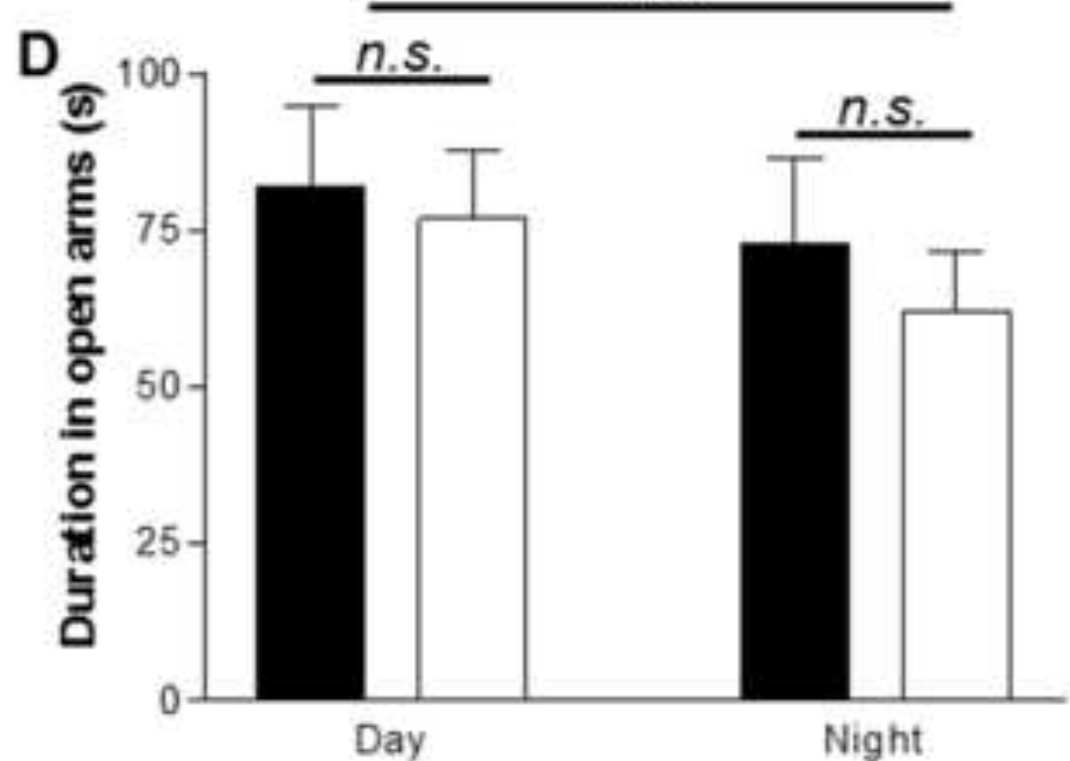


A

C
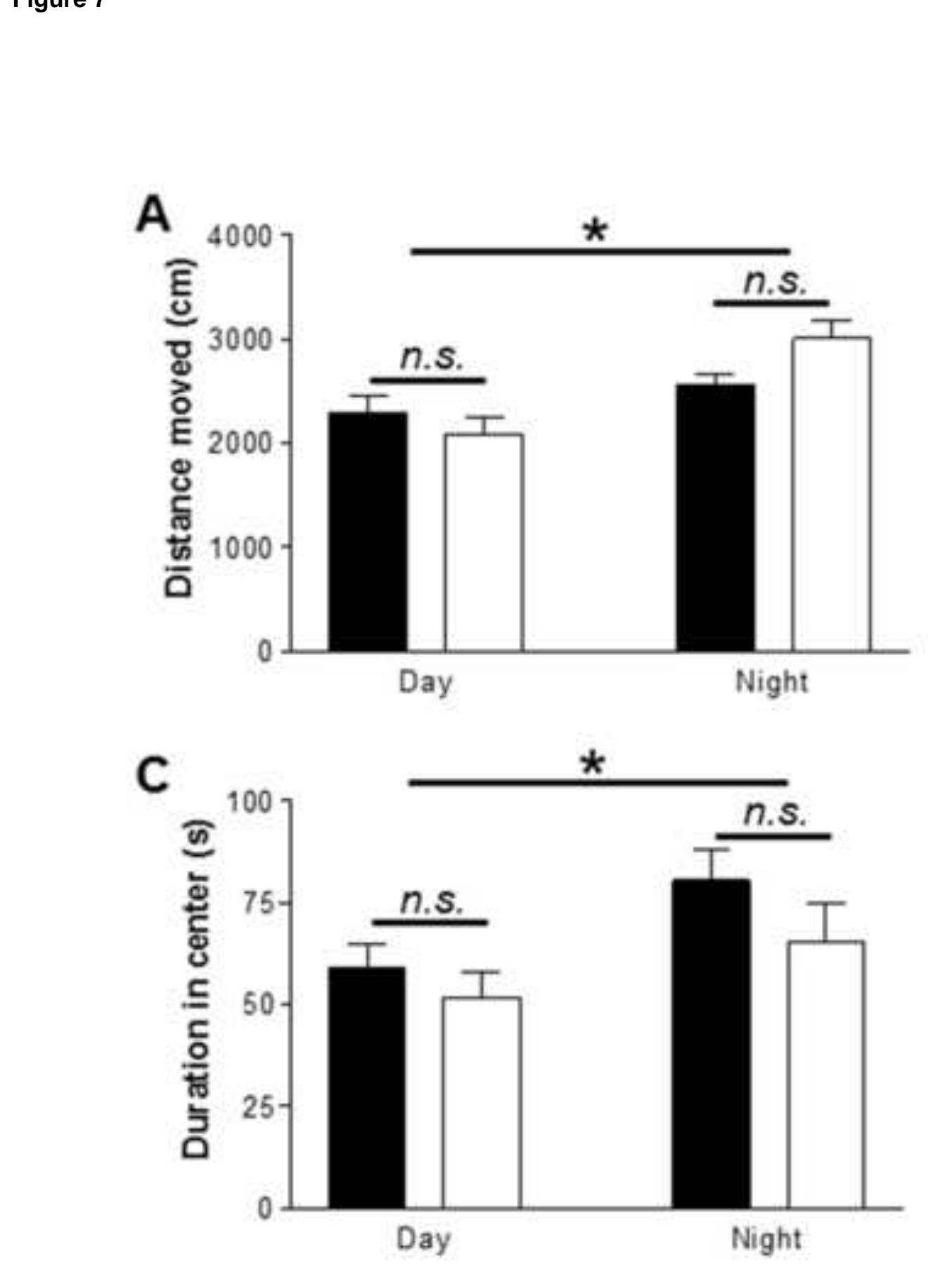

BMAL 1 WT
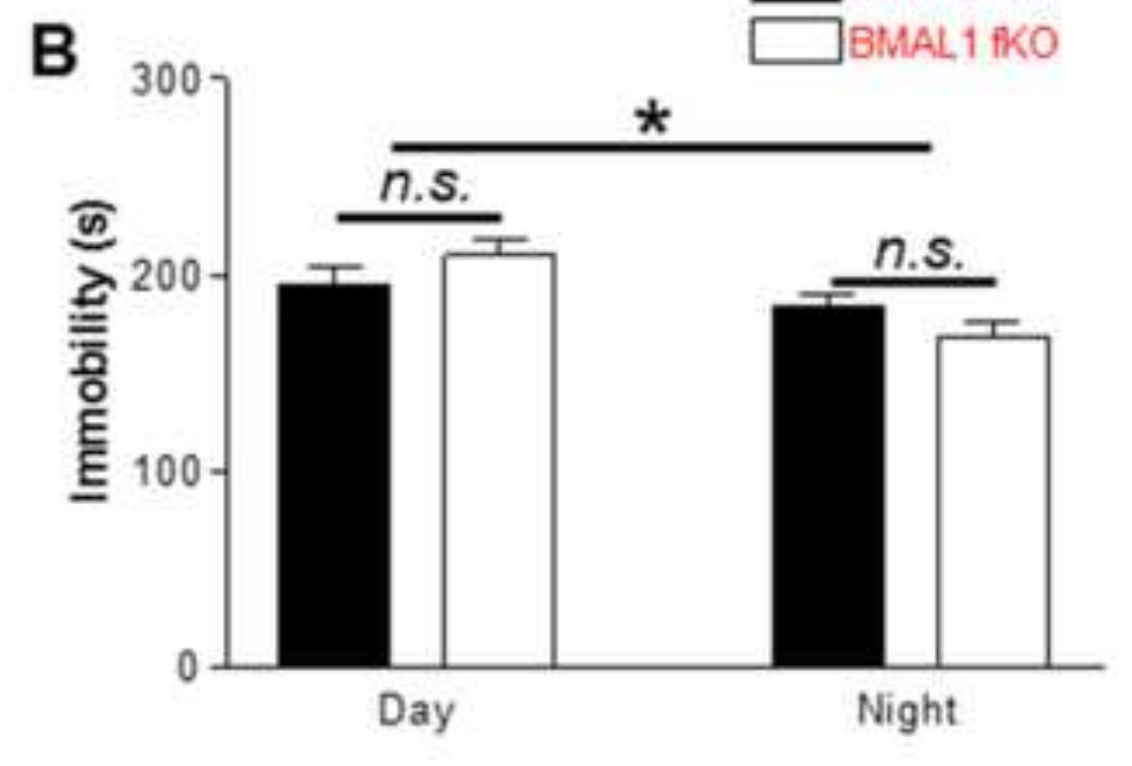

D

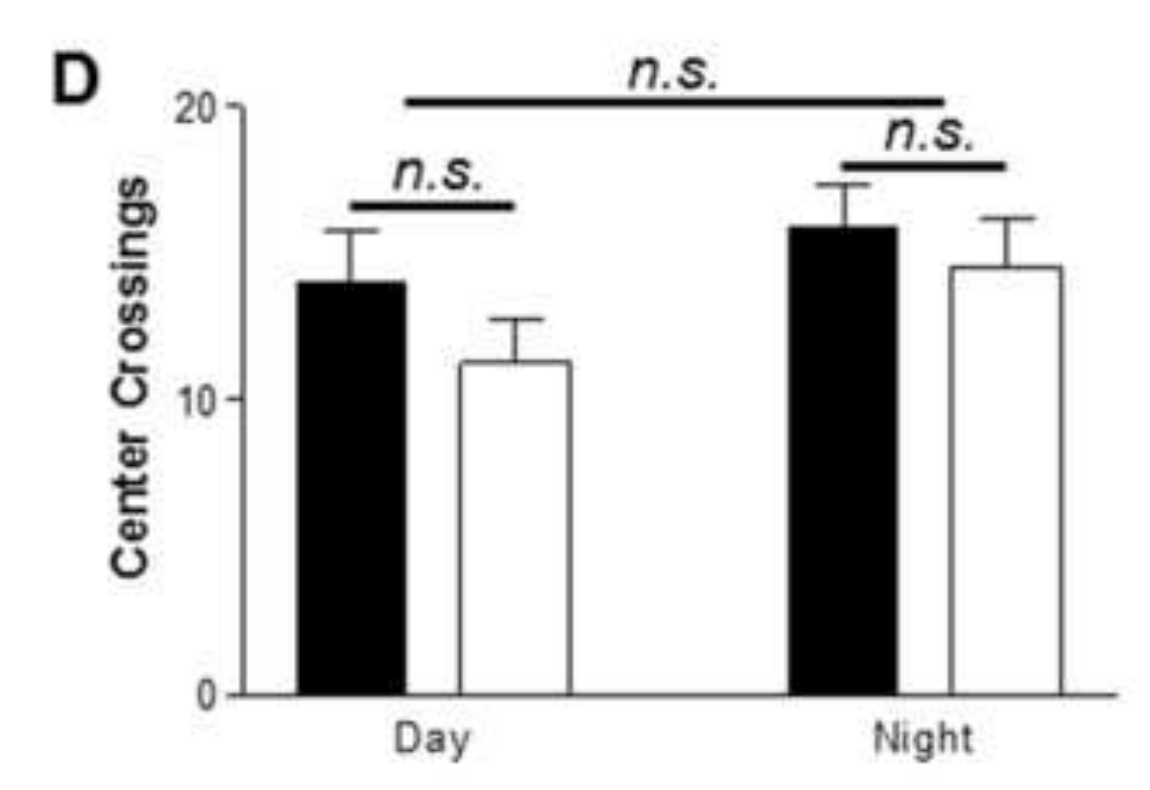




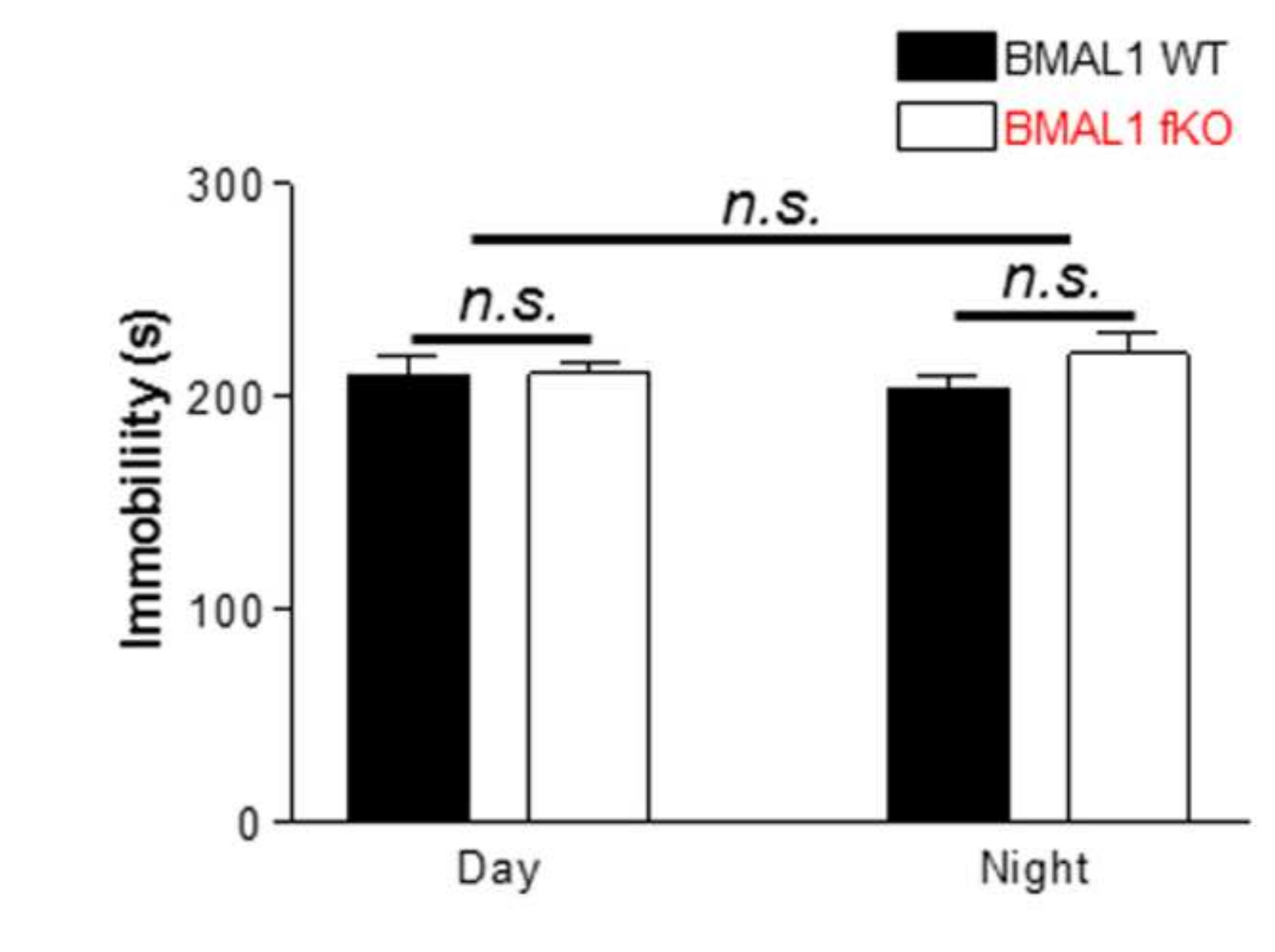

.

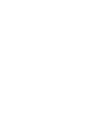
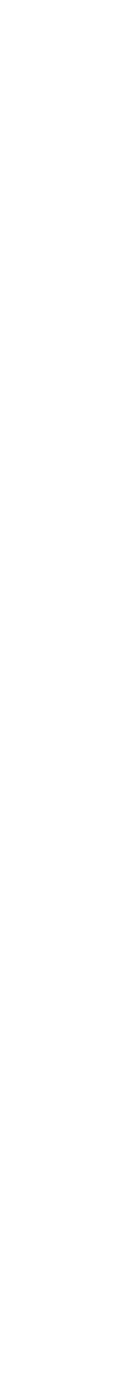